\title{
La experiencia de caminar en ciudades latinoamericanas
}

The experience of walking in Latin American cities

\section{Pablo Páramo}

Universidad Pedagógica Nacional. Bogotá (Colombia)

Andrea Burbano

Universidad Colegio Mayor de Cundinamarca. Bogotá (Colombia)

Miguel Ángel Aguilar

Universidad Autónoma Metropolitana. Ciudad de México (México)

Edgar García-Anco

Universidad Nacional de San Agustín de Arequipa. Arequipa (Perú)

Edward Silvestre Pari-Portillo

Universidad Nacional de San Agustín de Arequipa. Arequipa (Perú)

Páramo, P., Burbano, A., Aguilar, M., García-Anco, E., Pari-Portillo, E., Jiménez-Domínguez, B., López-Aguilar, R., Moyano-Díaz, E., Viera, E., Elgier, A. y Rosas, G. (202I). La experiencia del caminar en ciudades Latinoamericanas. Revista de Arquitectura (Bogotá), 23(I), 20-33. https://doi.org//0.14718/ RevArq.2021.2830

\section{(c) (1) (\$)}

\section{Pablo Páramo}

Psicólogo, Universidad Católica de Colombia, Bogotá (Colombia).

PhD, en Psicología, Centro de Graduados de la Universidad de la

Ciudad de Nueva York (Estados Unidos).

Master of Science, University of Surrey. Reino Unido.

Becario, Consejo Británico, 1993 y Becario Fulbright, 1999

-https://scholar.google.es/citations?user=rGq8GlwAAAAJ\&hl=es

http://scienti.colciencias.gov.co:808I/cvlac/visualizador/generarCu-

rriculoCv.do?cod rh $=0000315133$

(D) https://orcid.org/0000-0002-455I-3040

@10pparamo@pedagogica.edu.co,pdeparamo@gmail.com

\section{Andrea Burbano}

Arquitecta, Universidad de La Salle, Bogotá (Colombia).

PhD. en Estudios Territoriales, Universidad de Caldas, Manizales (Colombia)

Master en Gestión Urbana, Universidad Piloto de Colombia, Bogotá (Colombia).

https://scholar.google.es/citations?user=DsMthqMAAAA|\&hl=es

http://scienti.colciencias.gov.co:808I/cvlac/visualizador/generarCurriculoCv.do?cod_rh $=0001198653$

(iD) https://orcid.org/0000-0003-2037-5603

@amburbano@unicolmayor.edu.co,arqburbano@gmail.co

Miguel Ángel Aguilar

Psicólogo social, Universidad Autónoma Metropolitana, Ciudad de México (México).

Doctor en Ciencias Antropológicas, Universidad Autónoma Metropolitana, Ciudad de México (México)

(D) https://orcid.org/0000-0002-1694-7886

(9)mad@xanum.uam.mx,miguelangeluami@gmail.co

\section{Edgar García-Anco}

Arquitecto y urbanista, Universidad Nacional de San Agustín de Arequipa. Arequipa (Perú)

Doctorado en Arquitectura y Urbanismo con mención en Vivienda, UNSA 20II.

Master en Planificación y gestión urbana ambiental, Universidad Nacional de San Agustín de Arequipa. Arequipa (Perú).

(D) https://orcid.org/0000-00402-7534-2086

(10) egarciaan@unsa.edu.pe

Edward Silvestre Pari-Portillo

Arquitecto y urbanista, Universidad Nacional de San Agustín de Arequipa. Arequipa (Perú).

Master en ciencias con mención en planificación y gestión urbano ambiental, Universidad Nacional de San Agustín de Arequipa, Arequipa (Perú)

(i) https://orcid.org/0000-0003-3937-4002

@epari@unsa.edu.pe
Bernardo Jiménez-Domínguez

Universidad de Guadalajara. Guadalajara (México)

Rosa Margarita López-Aguilar

Universidad de Guadalajara. Guadalajara (México)

Emilio Moyano-Díaz

Universidad de Talca. Talca (Chile)

José Viera

Universidad de la República. Montevideo (Uruguay)

Ángel Manuel Elgier

Universidad Abierta Interamericana-Universidad de Buenos Aires. (Argentina)

Guillermo Rosas

Universidad Pedagógica y Tecnológica de Colombia. Tunja (Colombia)
Bernardo Jiménez-Domínguez

Psicólogo, Universidad de los Andes, Bogotá (Colombia)

Doctor en Psicología Social, Universidad Autónoma de Barcelona, Barcelona (España)

Master en Psicología Social, Universidad Autónoma de Barcelona, Barcelona (España)

(D) https://orcid.org/0000-0002-80|3-9|2X

@ bernardo.jimenez@academicos.udg.mx,bjimdom@hotmail. com

Rosa Margarita López-Aguilar

Licenciada en Psicología, Universidad de Guadalajara, Guadalajara (México)

Doctora en Ciencias Sociales con Especialidad en Antropología Social, Centro de Investigaciones y Estudios Superiores en Antropología Social (CIESAS) Unidad Occidente, Guadalajara (México)

(1D) https://orcid.org/0000-0003-0307-9669

@ rosa.laguilar@academicos.udg.mx,rosilopag@gmail.com

\section{Emilio Moyano-Díaz}

Psicólogo, Pontificia Universidad Católica de Chile, Santiago (Chile)

Doctor en Psicología, Universidad Católica de Lovaina, Lovaina la

Nueva (Bélgica)

(D) https://orcid.org/0000-0003-2433-927|

@10 emoyano@utalca.cl, eemoyano@gmail.com

José Viera

Licenciado en Psicología, Universidad de la República, Montevideo (Uruguay)

Doctor en Psicología, Universidad Nacional de San Luis, San Luis (Argentina)

Master en Ciencias Humanas, Estudios Latinoamericanos, Universidad de la República, Montevideo (Uruguay)

(1D) https://orcid.org/0000-0003-0670-4618

(10)eviera@psico.edu.uy,edujoviera@gmail.com

Ángel Manuel Elgier

Licenciado en Psicología, Universidad de Buenos Aires, Buenos Aires (Argentina)

Doctor en Psicología, Universidad Nacional de Córdoba, Córdoba

(Argentina)

(D) https://orcid.org/0000-0002-6II0-5I5

@ angel.elgier@uai.edu.ar, amelgier@gmail.com

\section{Guillermo Rosas}

Economista, Universidad Pedagógica y Tecnológica de Colombia, Tunja (Colombia)

(iD https://orcid.org/0000-000 I-9642-95 I

@ guillermo.rosas@uptc.edu.co, guillermo.rosas.romero@gmail.co 


\section{Resumen}

Se exponen los resultados de un estudio descriptivo que explora la forma como los habitantes de 8 ciudades de 6 países latinoamericanos (México, Colombia, Perú, Argentina, Uruguay y Chile) valoran las condiciones que las hacen caminables. Un total de 1133 personas calificaron, mediante un cuestionario conformado por 50 ítems, distintas situaciones que se presentan al caminar, instrumento que fue complementado por 144 entrevistas semiestructuradas. A partir de un análisis descriptivo correlacional, mediante el programa de escalamiento multidimensional, Smallest Space Analysis (SSA), se confirman las 3 dimensiones propuestas para recoger la experiencia del caminar: I) los propósitos que persiguen las personas al caminar, 2) el lugar por donde se camina y 3 ) las condiciones espaciales y sociales que inhiben o promueven esta experiencia; se identifican, además, los elementos que integran cada dimensión. Estos hallazgos se integran con los resultados de las entrevistas semiestructurada para ser discutidos en términos de sus implicaciones para la planeación y la gestión urbanas.

Palabras clave: Migración pendular; calidad de vida; espacio urbano; urbanización; ordenamiento urbano

\begin{abstract}
The results of a descriptive study that explores how the inhabitants of eight cities in six Latin American countries (Mexico, Colombia, Peru, Argentina, Uruguay and Chile) evaluate the conditions that make those cities walkable are presented. A total of I I 33 people rated, by means of a questionnaire made up of 50 items, different situations that arise when walking, an instrument that was complemented by 144 semi-structured interviews. Based on a descriptive correlational analysis, using the multidimensional scaling program, Smallest Space Analysis (SSA), the three dimensions proposed to capture the experience of walking are confirmed: I) the purposes that people pursue when walking, 2) the place where they walk, and 3) the spatial and social conditions that inhibit or promote this experience; the elements that make up each dimension are also identified. These findings are integrated with the results of the semi-structured interviews to be discussed in terms of their implications for urban planning and management.
\end{abstract}

Key words: Pendular migration; quality of life; urban space; urbanization; urban development; urban planning;

\section{Introducción}

El presente artículo, resultado de investigación, se enmarca dentro de la línea de investigación de espacio público que desarrolla el grupo de investigación Pedagogía Urbana y Ambiental de la Universidad Pedagógica Nacional, Bogotá, Colombia. El trabajo realizado da continuidad al proyecto de investigación El Caminar como Experiencia de Aprendizaje de la Ciudad, financiado por el Centro de Investigaciones de la mencionada universidad, y el cual tuvo como objetivo identificar la estructura conceptual mediante la que los participantes de varias ciudades de América Latina valoran las condiciones físicas y sociales que posibilitan la experiencia de caminar.
Es ampliamente conocida la importancia que le han dado poetas y novelistas a caminar por la ciudad. En su ensayo El hombre de la multitud (1840), el escritor Edgar Allan Poe caracteriza al urbanita de la modernidad como un ser anónimo que se camufla en la multitud que deambula por la ciudad. Por su parte, en El declive del hombre público (1977) Richard Sennett critica la vida urbana a partir de la muerte del espacio público en París, la desconfianza sobre el extraño y el aislamiento del individuo, como consecuencia de una nueva configuración espacial y de nuevos estilos de vida en la sociedad urbanizada. Y al ver Manhattan desde las alturas del World Trade Center o caminando por sus calles, Michel de Certeau (1984) compara caminar con las palabras que conforman un lenguaje, y lo define como un acto político de apropiación. Pero es solo hasta años recientes que se le ha dado importancia al caminar desde el urbanismo y los programas de salud y recreación.

Este artículo enfatiza, con una perspectiva urbanística, caminar en las ciudades latinoamericanas, a partir de distintas facetas o variables que se pueden visualizar de esta experiencia: como actividad física que contribuye a la salud; como mecanismo de movilidad para ir de un lugar a otro; como facilitador del encuentro social; como recreación y apreciación del paisaje natural y construido; como experiencia fenomenológica a través de la cual los individuos perciben el mundo y experimentan una serie de sensaciones, o tan simple como la manera de proveer bienestar a las mascotas. Beneficios adicionales de caminar se observan en una mejor calidad del aire, en la reducción de tráfico (Haines et al., 2009) y en mayor inclusión social. Pero también, y en un ámbito más general, se vienen analizando sus implicaciones en nociones tales como justicia social, ciudadanía, democracia, equidad y movilidad sostenible, lo que se enmarca dentro de la noción de "el derecho a la ciudad", planteado tempranamente por Lefebvre (1969) como parte activa de las luchas por una ciudad habitable, así como la reivindicación de la diversidad de formas de habitar el espacio público, ligado, a su vez, al concepto de la apropiación del espacio.

A pesar de su importancia para la salud, para la educación del ciudadano y para la experiencia emocional y social de sentirse parte de un conglomerado urbano, quienes planificaron y administraron la ciudad en el siglo XX no le pusieron suficiente atención a esta necesidad de las personas cuando se desplazan por el espacio público. Ello se refleja en la discontinuidad de las aceras, en su estrechez y en los pocos kilómetros que existen de ellas en buen estado, así como en la falta de luminarias en distintos sectores y la inseguridad percibida por los

This article is available in English on the website of Revista de Arquitectura (Bogotá) http://dx.doi.org/10.14718/RevArq.2021.2830

The experience of walking in Latin American cities 
habitantes, lo que puede explicarse a partir del modelo modernista de las grandes urbes estadounidenses de los años cincuenta y sesenta del siglo XX, en el que se privilegió la movilidad de los automóviles cuando las personas comenzaron a habitar los suburbios, y que fue seguido por varias ciudades latinoamericanas (Oldemburg, 2013), dejando como algo marginal el acto de caminar. Sumado a la copia de este modelo, los países latinoamericanos tuvieron que priorizar el déficit de vivienda, lo que limitó la planeación de espacios para caminar.

No obstante lo anterior, la nueva configuración social del siglo XXI, resultado de la globalización, la migración y la alta densidad poblacional en las ciudades, el desarrollo de la tecnología y el impacto de la contaminación atmosférica han impulsado en el mundo un interés por mejorar la calidad de vida urbana. En consecuencia, se han propuesto nuevos diseños urbanos, con medios alternativos a la movilidad motorizada, entre los cuales se destacan la promoción del uso de la bicicleta y caminar, que ahora hacen parte de los planes de rediseño urbano y de la intención de recuperar y ampliar los espacios públicos, si bien son escasos los estudios que toman en cuenta el acto de caminar.

Surge entonces la necesidad de explorar ahora las razones por las cuales la gente camina, o prefieren hacerlo, así como las situaciones que facilitan o restringen su caminar partiendo de las características del sitio por donde se camina, con el propósito de justificar ante quienes planifican y gestionan la ciudad la importancia de diseñar el espacio público pensando en el peatón.

La mayor parte de los estudios están asociados a los beneficios que tiene caminar como prevención de enfermedades o como rehabilitación de ellas (Bassett et al., 2008; Haines et al., 2009; Forjuoh et al., 2017; Keller y Cantue, 2008). En relación con su importancia para la recreación y el ejercicio físi$\mathrm{co}$, las investigaciones dan cuenta de la riqueza de promover una comprensión más amplia de estas actividades, tanto para la recreación como para la movilidad (Lee y Ingold, 2006; Le Breton, 2007; Keller y Cantue, 2008). Igualmente, caminar ha sido abordado desde su relación con la memoria y la afectividad urbana (Aguilar, 2016; 2018).

En lo que concierne a la seguridad, los trabajos auspiciados por la Fundación MAPFRE (2016) y el de Conejera et al. (2003) hacen ver que la morbilidad y la mortalidad de los peatones ocupa los primeros lugares en los países latinoamericanos. Existen reportes acerca de la intencionalidad de transgredir normas de tránsito al caminar por parte de adultos de ambos sexos (Moyano Díaz, 2002) así como diferencias según edad (Moyano Díaz, 1999). Cada año se registran, en promedio, 1,3 millones de muertes por accidentes de tránsito en el mundo, según datos de la Organización Mundial de la Salud (OMS), y según los cuales Latinoamérica es la región más afectada. Casi la mitad de estas muertes ocurren en las ciudades, y la mayoría de las víctimas son peatones. Así se alerta en un reciente informe de la OMS y la Organización Panamericana de la Salud (OPS) (2016). En consecuencia, algunas ciudades se vienen ocupando de proporcionar espacios públicos caminables y seguros. Barcelona y Nueva York, por ejemplo, han creado las supermanzanas, dentro de las cuales la movilidad vehicular está prohibida, por lo que caminar por dichas manzanas es más seguro. Por su parte, en Medellín, se han creado rutas seguras para los niños en edad preescolar y que viven en barrios con altos índices de violencia. Los niños caminan hacia la escuela acompañados de adultos, jugando y escuchando música.

Con la perspectiva de género, los estudios han evidenciado que caminar es arriesgado para las mujeres, no solo por las barreras espaciales — por ejemplo, cuando llevan un coche para un bebé-, sino por las barreras simbólicas que condicionan su acceso a los espacios públicos (Burbano, 2016a; 2014; Lindón, 2006; Falú, 2009). Para Ortiz (2007), las mujeres evitan ser agredidas y ser víctimas de asaltos y de acosos sexuales cuando caminan por el espacio público. Los peligros que enfrentan al caminar llevan a que naturalicen rutinas de movilidad con el fin de evitar el peligro optando por tomar rutas conocidas, modificar rutas y horarios para el desplazamiento o, simplemente, recluirse en el hogar (Burbano, 2014, 2016b).

A partir de la psicología ambiental y social en la que se indaga por la dimensión psicoespacial y su relación con el comportamiento, se viene haciendo un llamado de alerta sobre la privatización de los espacios públicos y su impacto sobre la socialización y la caminabilidad de las ciudades (Jacobs, 1961; Low, 2005; Páramo, 2011).

Al trazar su ruta para ir de un lugar a otro, las personas suelen revisar si hay aceras o no, si hay cruces demarcados para peatones y el tiempo del viaje (Hollenstein y Bleischa, 2016). D'Alessandro et al. (2016) Ilaman la atención sobre aspectos más subjetivos, como la seguridad percibida y la valoración que hacen las personas sobre la urbanidad de las personas con las que se suelen encontrar en las calles.

Entre los estudios de tipo comparado, tiene relevancia el de Kerr et al. (2016), en el que se comparan 17 ciudades en el mundo, de 12 países de distintos continentes, en relación con las condiciones que los individuos señalan como indispensables para movilizarse por el vecindario o la ciudad, ya sea que lo hagan en bicicleta o a pie. Se destacan la seguridad, la existencia de lugares recreativos, la estética de lo que puede apreciarse, la accesibilidad a los centros comerciales, la percepción sobre la distancia para llegar a un sitio y la existencia de paraderos de buses.

De acuerdo con Speck (2012), para que una ciudad sea caminable es necesario que las caminatas sean mejores, o al menos, iguales a los otros medios de movilidad, para lo cual es necesario que las caminatas reúnan las siguientes características: 1) que exista un propósito para caminar (la caminata útil), 2) que caminar sea seguro (la 
caminata segura) y 3) que sea agradable (la caminata agradable/confortable) e interesante.

Al analizar las políticas que promueven caminar en las ciudades, es necesario mencionar el impulso que le han venido dando distintas organizaciones internacionales, como la OMS y la OPS (OMS-OPS, 2016), la Fundación MAPFRE (2016), La Fundación Gonzalo Rodríguez (2018) y la Fundación para la Seguridad del Tráfico (AAA, 2018), entre otras, para promover la normatividad en los países de la región. En los documentos generados por estos organismos se hace hincapié en la importancia de caminar para la salud y la protección del ambiente, como parte de una aproximación integral que abarque disposiciones legislativas, además de técnicas y educativas, mediadas por la creación de leyes.

La Comisión Económica para América Latina y el Caribe (CEPAL, 2011), realiza acciones que promueven una mirada integral y multidisciplinaria sobre las políticas públicas de movilidad y el diseño de la infraestructura vial, y para promover la coordinación en áreas como educación, salud, normatividad, infraestructura, equipamiento y control, con el fin de lograr resultados efectivos y sostenidos en la reducción de la siniestralidad vial. Las influencias y las colaboraciones de la OMS-OPS (2016) y de la CEPAL (2011) se han reflejado en varios países en documentos técnicos que incorporan inversiones económicas para transformaciones urbanas $y$, de igual modo, estrategias educativas que incorporan al peatón. Es importante señalar, igualmente, a las organizaciones no gubernamentales (ONG) que incentivan caminar por razones de salud, recreativas, sociales y de seguridad. Por mencionar algunas: la Fundación Walk21, la Fundación Despacio, la Fundación Bernard Van Leer, la Fundación Colombiana de Caminantes y la Asociación Correcaminos, entre muchas otras (Torres, 2019). Cabe mencionar que junto a esta categoría de políticas internacionales surgen movimientos sociales que impulsan la creación y la aplicación de dichas políticas en las ciudades que se muestran socialmente, en espacios académicos como congresos, conferencias y distintas formas de encuentro, donde converge la socialización de los nuevos modos de ver las ciudades. Una de las más fuertes a escala mundial es la desarrollada por Walk21, organización benéfica internacional dedicada a apoyar y promover caminatas, para transformar la percepción de caminar en la ciudad, a través de políticas, programas y planificaciones efectivas en todo el mundo (Walk21, 2018).

En los estudios de gestión urbana existe el interés en diseñar indicadores e índices objetivos para evaluar y hacer seguimiento a las políticas públicas que implementan las ciudades mediante los cuales sea posible valorar aspectos como: la accesibilidad para el transporte urbano; la distancia a equipamientos, a servicios atractivos; la cantidad de metros lineales de ciclorrutas; el ancho y la longitud de los andenes o las plataformas peatonales; la existencia de rampas para peatones y discapacitados; la cantidad de luminarias, bancas y depósitos para la basura, y la distancia entre estas; el arbolado; las baterías sanitarias; la calidad del aire, y los puentes peatonales, entre otros (Talavera-García y Soria-Lara, 2015; Tribby et al., 2016; D'Alessandro et al., 2016; Gutiérrez-López et al., 2019).

Aun cuando las evaluaciones a partir de indicadores objetivos sobre el ambiente físico son importantes, las que hacen los individuos sobre su experiencia subjetiva de caminar aportan información importante. Situaciones como las que explora el presente estudio juegan un papel importante para identificar las necesidades de los ciudadanos y mejorar su calidad de vida urbana.

\section{Metodología}

\section{Diseño}

Estudio con metodologia mixta, correlacional-descriptivo, que compara ocho matrices de datos cuantitativos derivados de la aplicación de un cuestionario a partir de un análisis correlacional. Se complementa, así mismo, con entrevistas en profundidad. Para su desarrollo metodológico se siguió el mismo procedimiento llevado a cabo en otros estudios comparados realizados por parte de algunos de los autores del presente trabajo (Páramo y Burbano, 2019; Páramo et al., 2018).

\section{Participantes}

Mediante un muestreo no probabilístico intencional, participaron voluntariamente 1277 personas habitantes de 11 ciudades latinoamericanas: Arequipa (Perú), Bogotá y Tunja (Colombia), Buenos Aires (Argentina), Ciudad de México y Guadalajara (México), Montevideo (Uruguay) y Talca (Chile). Del total de participantes, 1133 diligenciaron un cuestionario. La muestra fue categorizable en 4 rangos de edad. El 57,3\% de los participantes son mujeres $(n=650)$, el $42,5 \%$ son hombres $(n=481)$ y el $0,2 \%(n=2)$ informaron pertenecer a otro género, como se muestra en la tabla 1 . Del total de participantes, 144 respondieron a las entrevistas, como se muestra en la tabla 2.

\section{Instrumentos}

Se diseñó un cuestionario compuesto por 49 ítems, que aluden a qué tan caminable es la ciudad para los participantes, desde 3 dimensiones: 1) el propósito que persigue la persona cuando sale a caminar, 2) el lugar por donde camina y 3) las condiciones socioespaciales que facilitan o impiden caminar, partiendo de una escala de 7 puntos: desde muy poco caminable (1) a muy caminable (7). El ítem 49 indagaba por si las mujeres se sienten acosadas cuando caminan, por lo que debía ser respondido únicamente por las personas que se identifican con esta condición de género. Se incluyó un ítem adicional (ítem 50), que preguntaba por la caminabilidad en general de la ciudad. La escala de valoración incluyó la opción de respuesta "No sabe", para 


\begin{tabular}{|c|c|c|c|c|c|c|c|c|c|}
\hline \multirow[b]{2}{*}{ Ciudad } & \multicolumn{5}{|c|}{ Edad } & \multicolumn{3}{|c|}{ Género } & \multirow[t]{2}{*}{ Total } \\
\hline & 18-30 años & 31-45 años & 46-60 años & $\begin{array}{l}61 \text { años en } \\
\text { adelante }\end{array}$ & $\begin{array}{c}\text { Sin } \\
\text { información } \\
\text { de edad }\end{array}$ & Femenino & Masculino & Otro & \\
\hline Arequipa & 76 & 51 & 47 & 4 & 0 & 89 & 89 & 0 & 178 \\
\hline Bogotá & 173 & 93 & 24 & 15 & 0 & 164 & 141 & 0 & 305 \\
\hline Buenos Aires & 46 & 31 & 7 & 1 & 0 & 64 & 21 & 0 & 85 \\
\hline Ciudad de México & 69 & 31 & 22 & 11 & 0 & 72 & 60 & 1 & 133 \\
\hline Guadalajara & 132 & 14 & 11 & 2 & 0 & 105 & 53 & 1 & 159 \\
\hline Montevideo & 42 & 62 & 18 & 7 & 0 & 73 & 56 & 0 & 129 \\
\hline Talca & 45 & 6 & 6 & 1 & 0 & 26 & 27 & 0 & 53 \\
\hline Tunja & 58 & 24 & 7 & 2 & 0 & 57 & 34 & 0 & 91 \\
\hline Total & 641 & 312 & 137 & 43 & 0 & 650 & 481 & 2 & 1133 \\
\hline Porcentaje & 56,6 & 27,5 & 12,1 & 3,8 & 0 & 57,3 & 42,5 & 0,2 & 100 \\
\hline
\end{tabular}

(4) Tabla 1. Distribución de la muestra de participantes que diligenciaron el cuestionario en diferentes ciudades, según edad y género.

Fuente: elaboración propia (2019). CC BY-NC

\begin{tabular}{|c|c|c|c|c|c|c|c|c|c|}
\hline \multirow[b]{2}{*}{ Ciudad } & \multicolumn{5}{|c|}{ Edad } & \multicolumn{3}{|c|}{ Género } & \multirow[t]{2}{*}{ Total } \\
\hline & $\begin{array}{c}18-30 \\
\text { años }\end{array}$ & $\begin{array}{c}31-45 \\
\text { años }\end{array}$ & $\begin{array}{c}46-60 \\
\text { años }\end{array}$ & $\begin{array}{l}61 \text { años en } \\
\text { adelante }\end{array}$ & $\begin{array}{c}\text { Sin } \\
\text { Información } \\
\text { de edad }\end{array}$ & Femenino & Masculino & Otro & \\
\hline Arequipa & 17 & 3 & 5 & 2 & 0 & 10 & 17 & 0 & 27 \\
\hline Bogotá & 15 & 7 & 3 & 4 & 0 & 16 & 13 & 0 & 29 \\
\hline Buenos Aires & 1 & 0 & 0 & 0 & 3 & 2 & 2 & 0 & 4 \\
\hline Ciudad de México & 9 & 5 & 6 & 2 & 0 & 11 & 11 & 0 & 22 \\
\hline Guadalajara & 18 & 2 & 4 & 0 & 0 & 15 & 9 & 0 & 24 \\
\hline Montevideo & 2 & 8 & 3 & 4 & 0 & 5 & 12 & 0 & 17 \\
\hline Santiago & 10 & 5 & 1 & 2 & 0 & 6 & 12 & 0 & 18 \\
\hline Temuco & 0 & 0 & 0 & 0 & 2 & 2 & 0 & 0 & 2 \\
\hline $\begin{array}{l}\text { Casos que reporta } \\
\text { información } \\
\text { incompleta }\end{array}$ & - & - & - & - & 5 & - & - & - & - \\
\hline Total & 72 & 30 & 22 & 14 & 5 & 67 & 76 & 0 & 143 \\
\hline Porcentaje & 50,3 & 21 & 15,4 & 9,8 & 3,5 & 46,8 & 53,2 & 0 & 100 \\
\hline
\end{tabular}

(4) Tabla 2. Distribución de los los casos donde la situación evaluada no aplica, participantes de las entrevistas según lo estimara el participante. Los ítems se a profundidad, de acuerdo con la ciudad, el género y el grupo de edad.

Fuente: elaboración propia (2019). CC BY-NC. según lo estimara el participante. Los ítems se
derivaron de la revisión de otros instrumentos identificados en la literatura sobre las condiciones que hacen caminables las ciudades siguiendo el modelo de teoría de facetas (Borg y Shye, 2005; Hackett, 2014). En la parte final del cuestionario se solicitó información sobre las características sociodemográficas del encuestado, la edad, el género y la localidad, el barrio o la comuna en que vive. El instrumento fue validado por 3 jueces independientes y mediante un estudio piloto llevado a cabo con 60 participantes. En cada país se hizo el ajuste de la terminología. El análisis de la consistencia interna del instrumento mediante la prueba Alfa de Cronbach fluctuó entre 0,89 y 0,96 entre las ciudades, lo cual refleja una buena fiabilidad del cuestionario. Además, se utilizó una entrevista semiestructurada que indagaba por las mismas dimensiones, con base en las siguientes preguntas: 1) ¿En qué momento del día o de la semana lo hace, y con qué propósitos?, 2) En los desplazamientos que me ha contado, żqué hace mientras camina? ¿En qué piensa?, 3) ¿Por dónde suele caminar? ¿Lo hace generalmente solo o acompañado?, 4) ¿Qué siente, huele, escucha o toca?, 5) ¿Qué suele ver a su alrededor?, 6) ¿Qué suele llevar consigo?, 7) ¿Hay algún rumbo o ruta en particular que le guste tomar cuando sale a caminar?, 8) Cuando camina a un mismo destino, ¿suele cambiar de ruta, o siempre sigue la misma?, 9) ¿Qué es lo que más disfruta de caminar, y lo que menos disfruta?, 10) ¿Cuál ha sido su experiencia de caminar cuando sale de la ciudad?, 11) ¿Qué dificultades encuentra al caminar por los espacios públicos?, 12) ¿Qué recuerda de su infancia y su adolescencia cuando caminaba por la ciudad?

\section{Procedimiento}

Tanto el cuestionario como la entrevista se aplicaron bajo la supervisión de los equipos de investigación de cada país, y se obtuvo el consentimiento informado de los participantes de cada ciudad asegurándoles a los participantes que la información recogida sería confidencial. 
No. Media DS

$2 \quad 4,28 \quad 0,54$

$3 \quad 4,86 \quad 0,48$

$4 \quad 5,00 \quad 0,30$

$5 \quad 4,30 \quad 0,65$

$\begin{array}{lll}6 & 4,24 & 0,37\end{array}$

$\begin{array}{lll}7 & 3,74 & 0,53\end{array}$

$8 \quad 4,28 \quad 0,41$

$9 \quad 3,83 \quad 0,59$

$10 \quad 5,06 \quad 0,49$

$11 \quad 4,49 \quad 0,38$

$124,23 \quad 0,39$

$13 \quad 3,98 \quad 0,39$

$14 \quad 3,90 \quad 0,29$

$15 \quad 4,08 \quad 0,45$

$16 \quad 3,03 \quad 0,71$

$17 \quad 5,01 \quad 0,50$

$18 \quad 3,59 \quad 0,75$

$19 \quad 4,25 \quad 0,52$

$20 \quad 4,38 \quad 0,48$

$21 \quad 3,89 \quad 0,47$

$22 \quad 3,94 \quad 0,42$

$23 \quad 4,16 \quad 0,45$

$24 \quad 3,83 \quad 0,34$

$25 \quad 3,64 \quad 0,54$

$26 \quad 3,62 \quad 0,56$

$27 \quad 3,94 \quad 0,50$

$28 \quad 3,27 \quad 1,04$

$29 \quad 3,89 \quad 0,23$

$30 \quad 3,57 \quad 0,64$

$31 \quad 4,29 \quad 0,36$

$32 \quad 3,24 \quad 1,59$

$33 \quad 3,17 \quad 0,77$

$34 \quad 4,08 \quad 0,42$

$35 \quad 4,36 \quad 0,51$

$36 \quad 3,18 \quad 0,98$

$\begin{array}{lll}37 & 3,82 & 0,87\end{array}$

$38 \quad 3,89 \quad 0,48$

$39 \quad 4,39 \quad 0,47$

$40 \quad 4,27 \quad 0,54$

$41 \quad 4,02 \quad 0,36$

$42 \quad 4,15 \quad 0,63$

$43 \quad 3,85 \quad 0,32$

$44 \quad 3,16 \quad 0,46$

$45 \quad 3,86 \quad 0,47$

$46 \quad 3,67 \quad 0,89$

$47 \quad 3,78 \quad 0,21$

$48 \quad 3,98 \quad 0,23$
Ítems

Evitar la congestión vehicular de las calles que produce el ruido de los automotores

Hacer ejercicio en el barrio, de forma segura

Sacar a pasear mi mascota en el barrio, gracias a que sigo las normas de convivencia

Hacer compras en el centro comercial con mi familia, independientemente del clima

Desplazarme a mi trabajo por las calles de la ciudad entre vendedores ambulantes

Conocer un sitio histórico de la ciudad, guiado por la señalización existente

Salir de mi universidad en horas de la noche con la iluminación existente en las calles

Mejorar mi condición de salud caminando por las aceras del barrio

Ahorrarme lo del pasaje para ir a mi trabajo/lugar de estudio bajo las condiciones climáticas de la ciudad

Ejercitarme de manera segura en un gimnasio dentro de un centro comercial/shopping

Conversar con amigos del barrio disfrutando de la arquitectura del entorno urbano

Divertirme con mi familia gracias a la disposición del mobiliario urbano

"Despejar mi cabeza" en la calle con muchas personas a mi alrededor

Relajarme del trabajo en el parque, pero libre de excrementos de perros

Tratar de conocer sitios de interés en la ciudad caminando por lugares donde hay grafitis

Regresar a mi casa por calles solitarias

Distraerme de forma segura con amigos en el centro comercial/shopping

Prevenir enfermedades gracias a la calidad del aire de esta ciudad

Hacer actividad física bajo las condiciones climáticas que brindan los ambientes naturales

Apreciar la arquitectura de los edificios del barrio

Hacer contacto con desconocidos gracias a las aceras/andenes/veredas que se encuentran por mi barrio

Desplazarme a mi lugar de trabajo/estudio por los puentes peatonales que hay en la ciudad.

Distraerme si encuentro avisos prohibitivos y cámaras de seguridad por el centro comercial/shopping

Hacer actividad física por el barrio teniendo en cuenta la continuidad existente de las aceras

Ahorrarme el dinero del pasaje esquivando los vehículos que cruzan las avenidas

Desplazarme a la casa por la vía exclusiva para bicicletas después del trabajo/estudio, para ahorrar lo del pasaje

Ahorrarme el dinero del pasaje caminando hacia mi lugar de estudio/trabajo con un buen clima

Pasar por una zona oscura porque voy tarde para el trabajo/estudio

Transitar por un parque para evitar la congestión vehicular

Pasar por una calle llena de basura porque la congestión del transporte público va a hacer que llegue tarde a mi lugar de trabajo/estudio

Movilizarme dentro del centro comercial haciendo uso del teléfono celular

Encontrar un dispensador de agua en calles y parques

Encontrar un policía a quien pueda pedir orientación para llegar a un lugar

Desplazarme a mi trabajo/estudio entre los habitantes de calle

Saber de las trasformaciones arquitectónicas de parques, plazas y edificios

Satisfacer una necesidad fisiológica en algún baño público de la ciudad

Entretenerme en la noche con cafés, restaurantes o exhibiciones de espectáculos artísticos gratuitos en plazas o parques

Interactuar con elementos naturales como ríos, lagos, jardines, y alamedas en la ciudad

Satisfacer mi curiosidad por los nuevos centros comerciales/shopping

Educar a las personas mediante señalización en lugares públicos

Hacer distintos tipos de trámites personales gracias a la conectividad de las aceras entre los distintos lugares del barrio

Desplazarme de manera segura por los ejes peatonales paralelos a la vía exclusiva para bicicleta

Hacer uso de rampas para que una persona con discapacidad pueda desplazarse por la ciudad

Que un invidente pueda cruzar avenidas con la ayuda de semáforos auditivos

Divertirme en lugares públicos en ausencia de malos olores

Interactuar con la tecnología en el espacio público con pantallas interactivas e internet

Entrar en contacto con lugares de turismo ecológico y contemplar la naturaleza

Poder asistir a eventos culturales en el centro comercial, lejos de la congestión vehicular
¿ Tabla 3. Medias y desviaciones estándar de los ítems acerca de caminar por la ciudad.

Fuente: elaboración propia (2019). CC BY-NC. 


\section{Resultados}

Con el fin de comparar las evaluaciones que hicieron los participantes sobre qué tan caminables son sus ciudades en términos de las distintas condiciones que se les presentaron, se compararon los valores de las medias para cada ítem como se muestra en la tabla 3. En general, se observa que las condiciones evaluadas en la escala de caminabilidad (1 a 7), fueron calificadas dentro del rango entre medios y altos. Con valores altos (promedio por encima de 5) están los ítems 10, 17 y 4, que están relacionados de manera indirecta con la seguridad, mientras que el ítem 16 obtuvo el promedio más bajo, de 3,03, y el cual se refiere direc- tamente a la seguridad percibida por las mujeres cuando caminan.

\section{Análisis de distancias mínimas (WSSA1)}

A fin de encontrar la estructura conceptual que subyace a la valoración de las distintas condiciones asociadas a las dimensiones propuestas para explorar la caminabilidad de las ciudades, los valores asignados a los distintos ítems fueron procesados mediante un análisis de distancias mínimas, o Smallest Space Analysis (Borg y Groenen, 2005), a través del programa Hudap ${ }^{\circledR}$ (Amar, 2001). El WSSA1 —Smallest Space Analysis- correlaciona los ítems mediante el cálculo
๑ Figura 1. Propósitos de los participantes cuando caminan.

Nota: adaptado de La caminabilidad en Bogotá: Propósitos y condiciones socio-espaciales que facilitan y limitan esa experiencia.

Fuente: Páramo y Burbano (2019, p. 18), Revista de Arquitectura (Bogotá), 21(2). CC BY-NC.
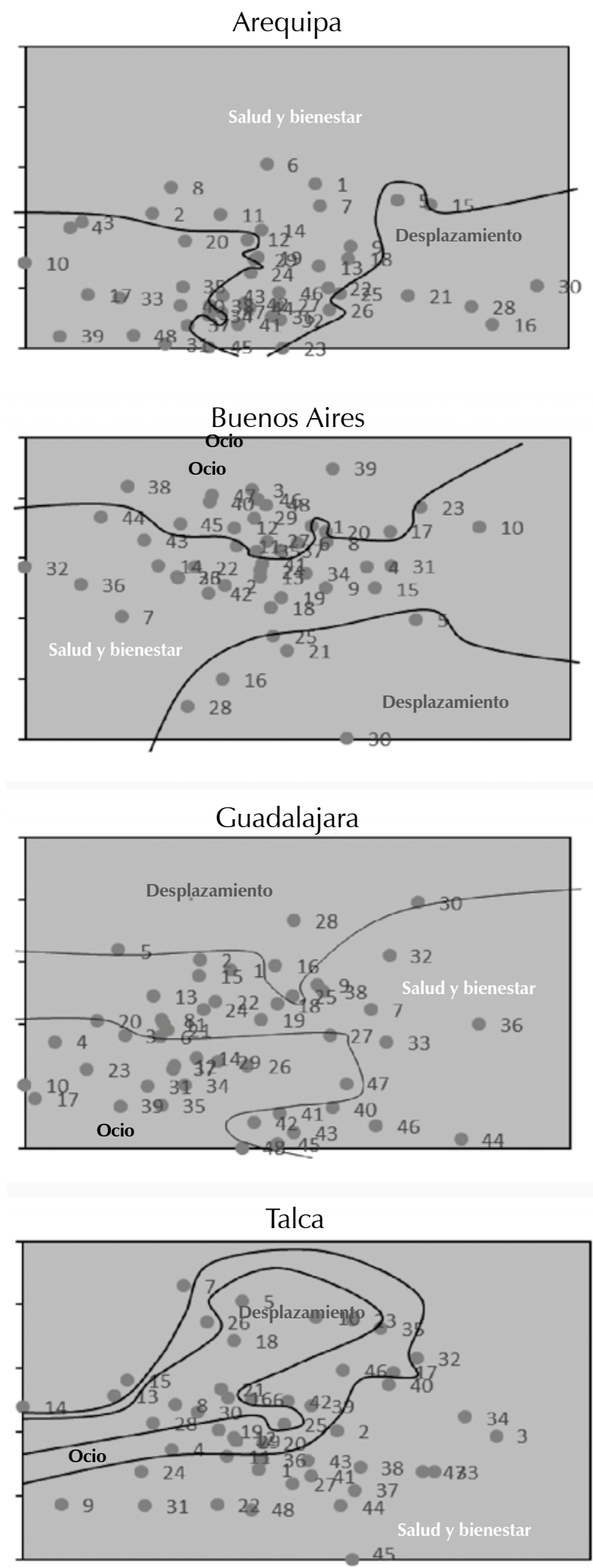

Bogotá

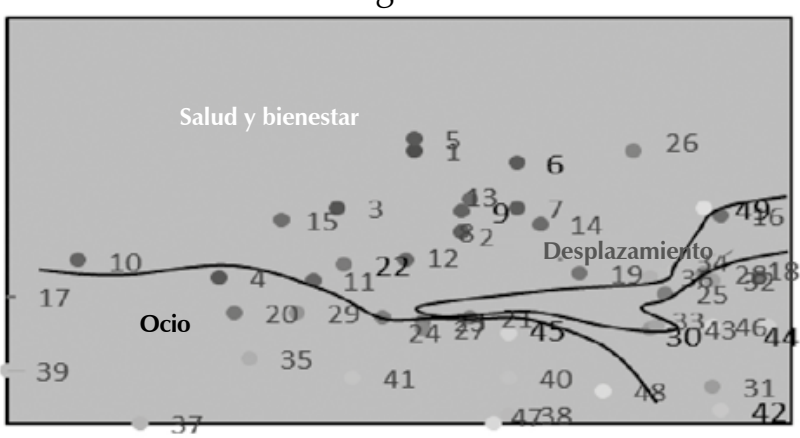

\section{Ciudad de México}
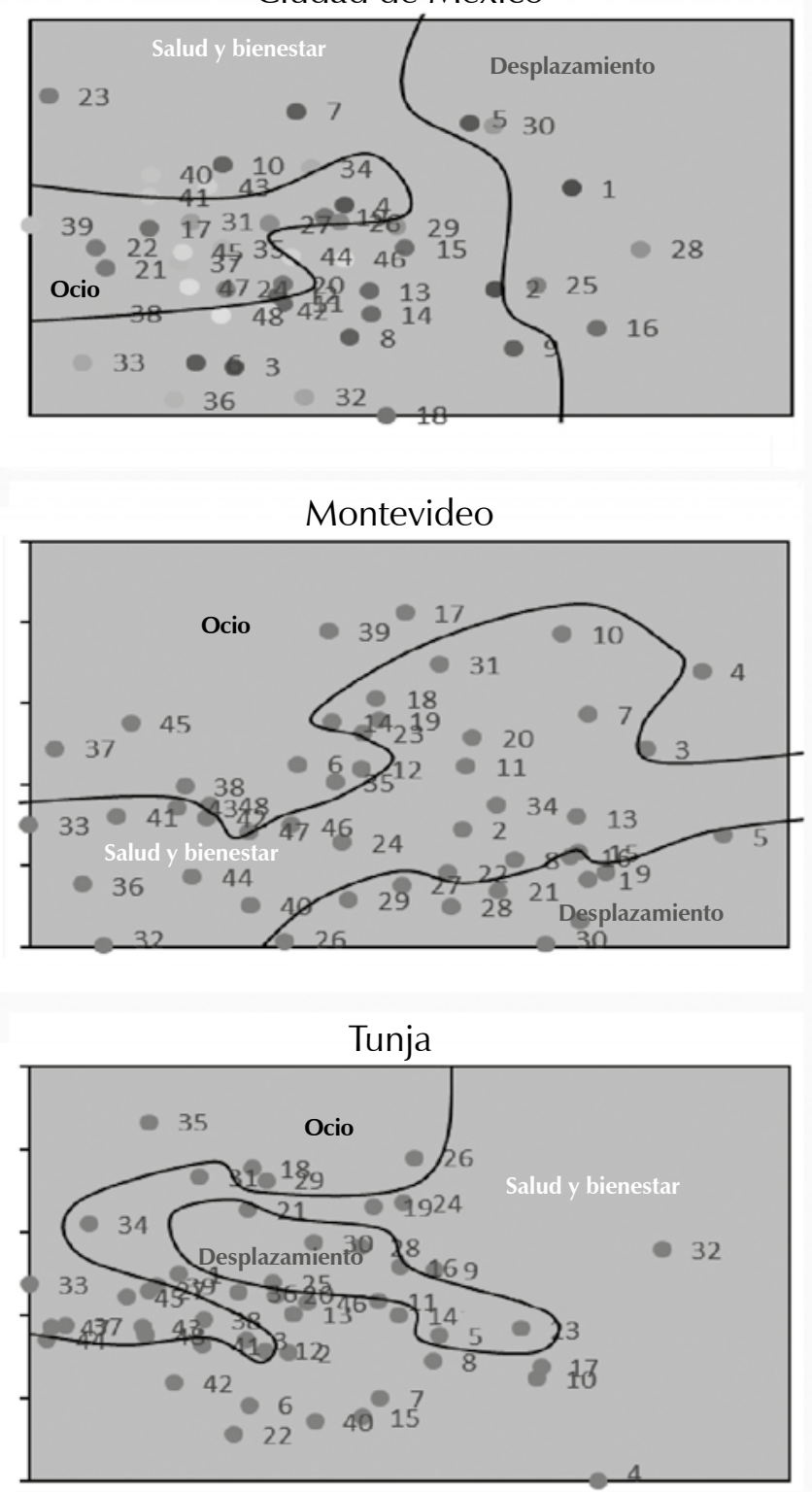
del índice de Pearson ${ }^{1}$. Las distancias espaciales entre los ítems, las cuales reflejan el grado de correlación entre estos, sirvieron de base para crear regiones o agrupaciones en los respectivos planos para cada una de las tres dimensiones propuestas. Este tipo de análisis es el que se utiliza en la teoría de facetas para estudiar diver-

1 Los resultados de la correlación de Pearson se muestran dentro de un plano que permite observar los ítems como puntos geométricos, de tal forma que mientras más similares en términos de la correlación entre ellos, más cerca se encontrarán espacialmente. Para llevar a cabo este análisis se crearon matrices independientes para las escalas de importancia y satisfacción del cuestionario.

Arequipa

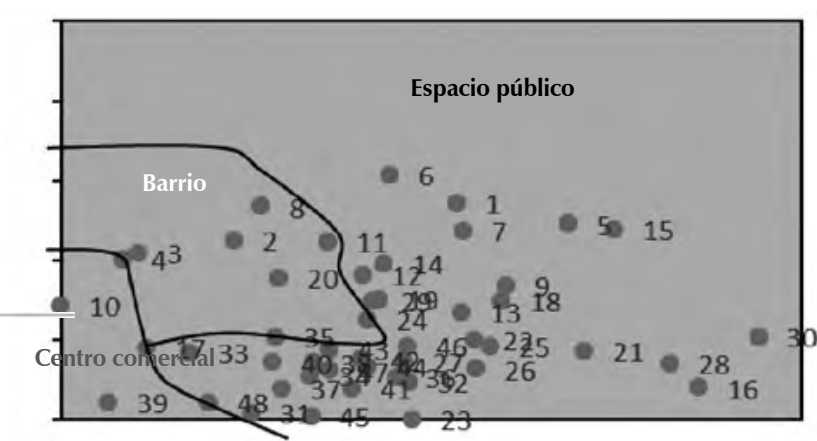

Buenos Aires

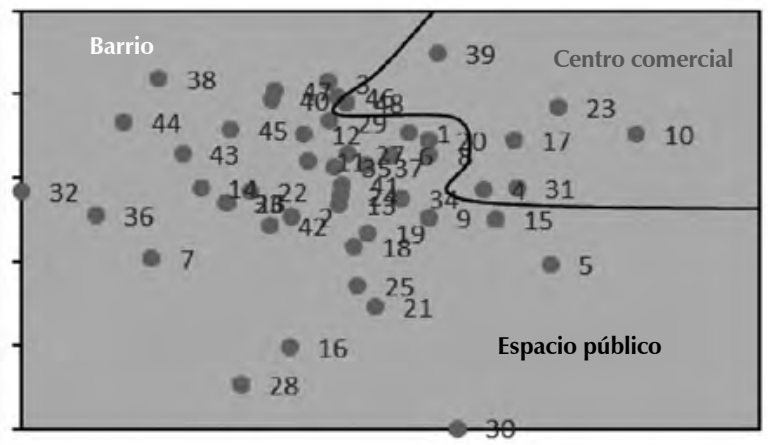

Guadalajara

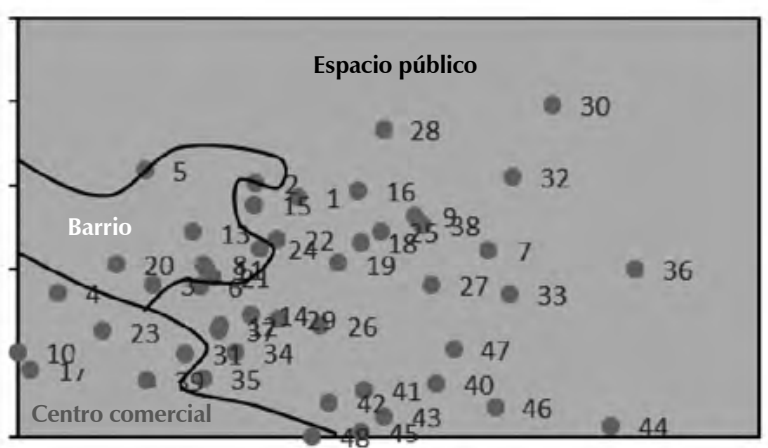

Talca

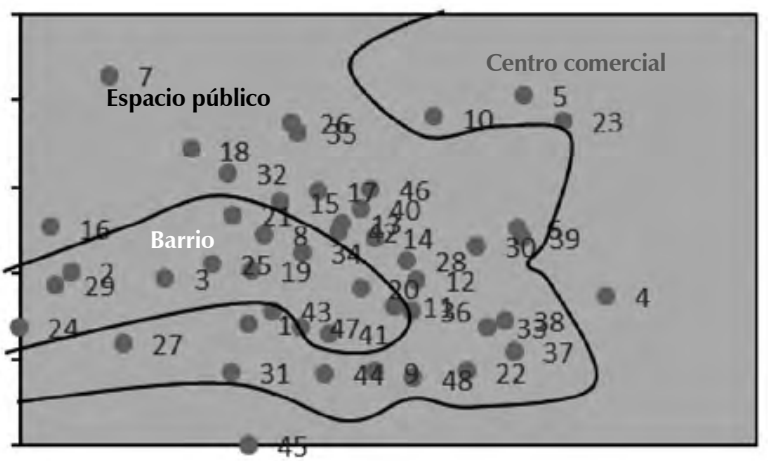

sos fenómenos sociales (Hackett, 2016), y que ha servido de base, igualmente, para el trabajo de Páramo y Burbano publicado por esta misma revista (2019).

De esa manera, para la dimensión "Propósitos", los ítems se agruparon en tres regiones, a partir de su cercanía espacial, lo que, a la vez, representa el grado de correlación entre ellos. Las regiones creadas a partir de la cercanía espacial y del contenido de los ítems dieron lugar a tres regiones: 1) salud y bienestar, 2) ocio y 3) desplazamiento, como se muestra en la figura 1. Para la dimensión "Lugar por donde se camina", los ítems se agruparon en tres regiones, siguiendo el mismo criterio

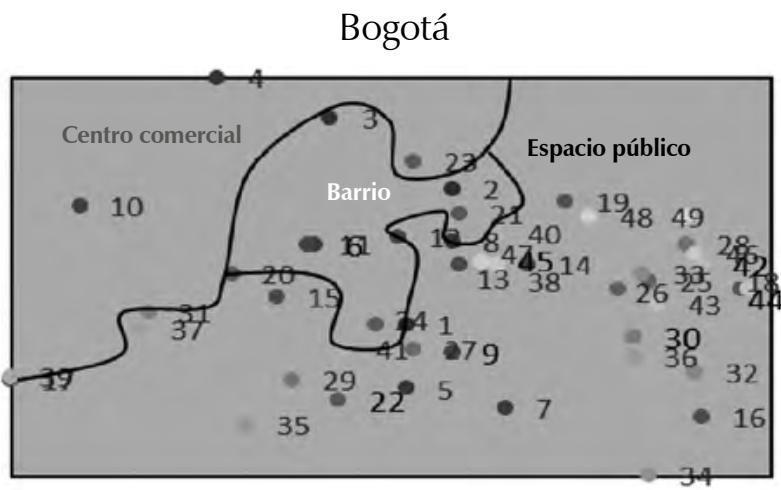

- Figura 2. Lugar por donde los participantes caminan. Nota: adaptado de La caminabilidad en Bogotá: Propósitos y condiciones socio-espaciales que facilitan y limitan esa experiencia. Fuente: Páramo y Burbano (2019, p.18), Revista de Arquitectura (Bogotá), 21(2). CC BY-NC.
Ciudad de México

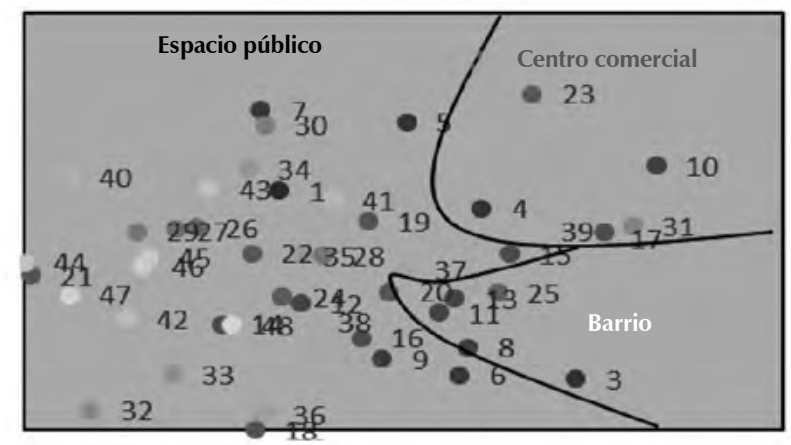

Montevideo

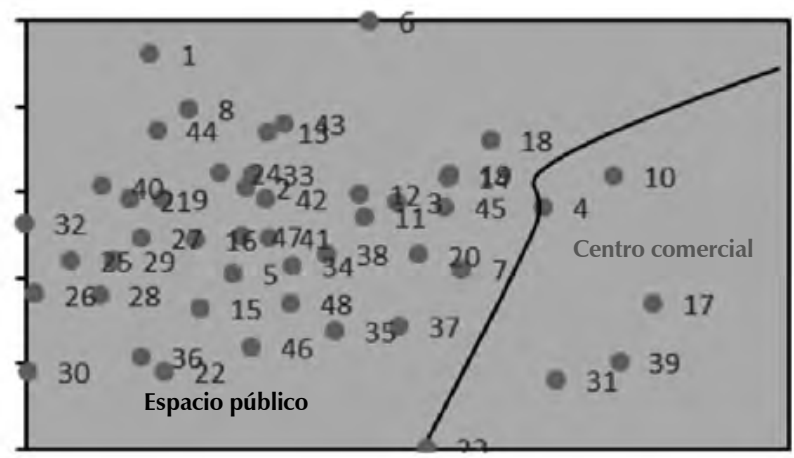

Tunja

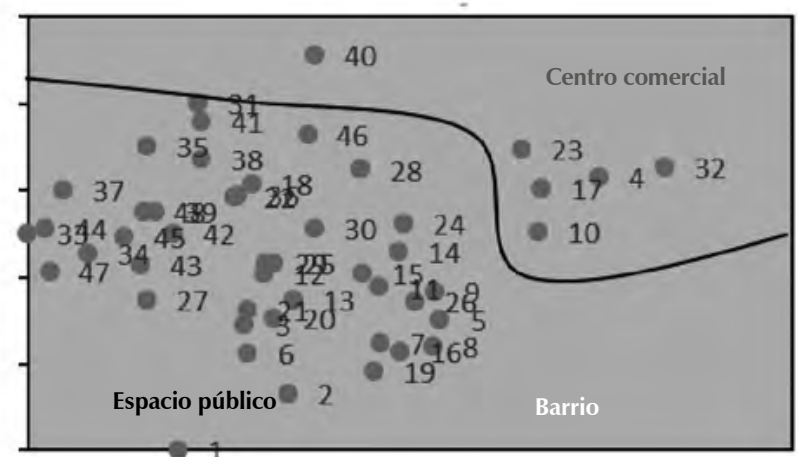


de cercanía espacial y el contenido de estos: 1 ) centro comercial, 2) espacios públicos naturales y construidos y 3) barrio, como se muestra en la figura 2, aunque no fue así para ciudades como Montevideo (Uruguay) y Tunja (Colombia), por cuanto los ítems que se refieren a los espacios públicos con los del barrio se observan fusionados, lo cual permite suponer que la representación de barrio no está claramente representada en los habitantes de estas ciudades (obsérvense las secciones de la figura que corresponde a estas

Arequipa

๑ Figura 3. Valoración de las condiciones sociales $y$ espaciales por donde se caminar.

Nota: adaptado de La caminabilidad en Bogotá: Propósitos y condiciones socio-espaciales que facilitan y limitan esa experiencia. Fuente: Páramo y Burbano (2019 p.19), Revista de Arquitectura (Bogotá), 21(2). CC BY-NC.

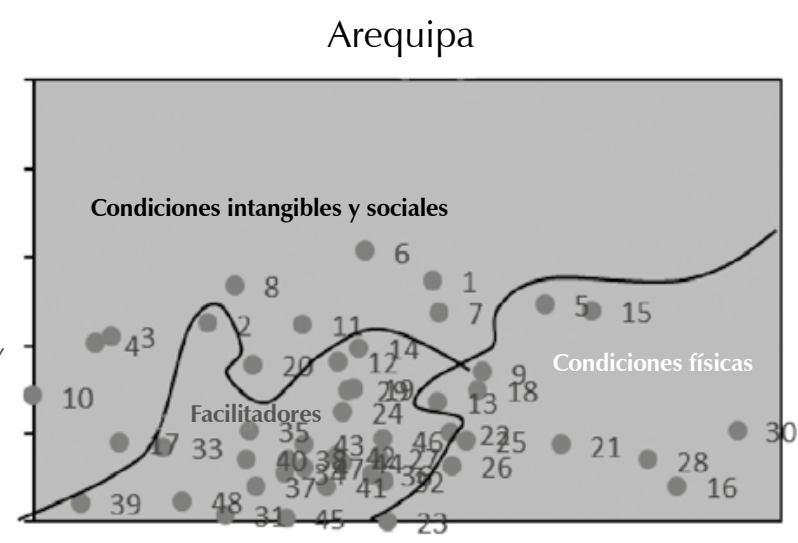

Buenos Aires

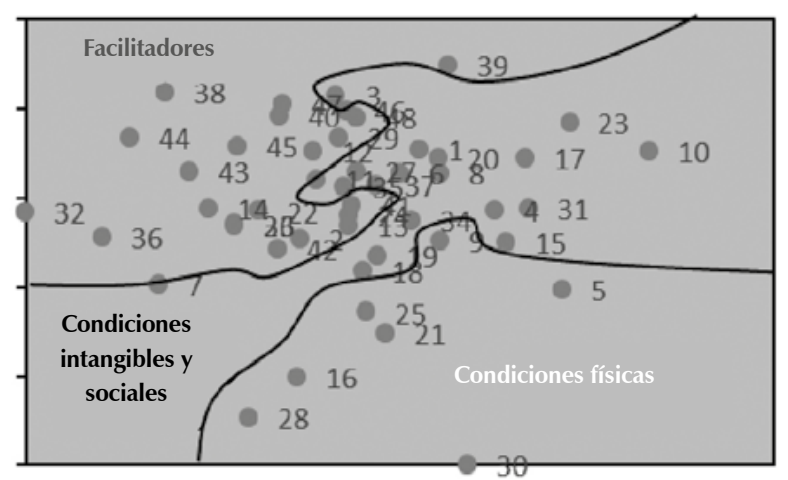

Guadalajara

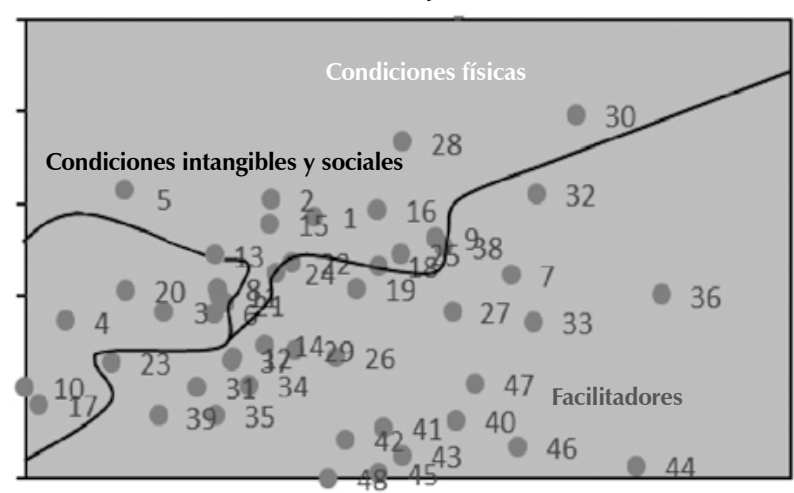

Talca

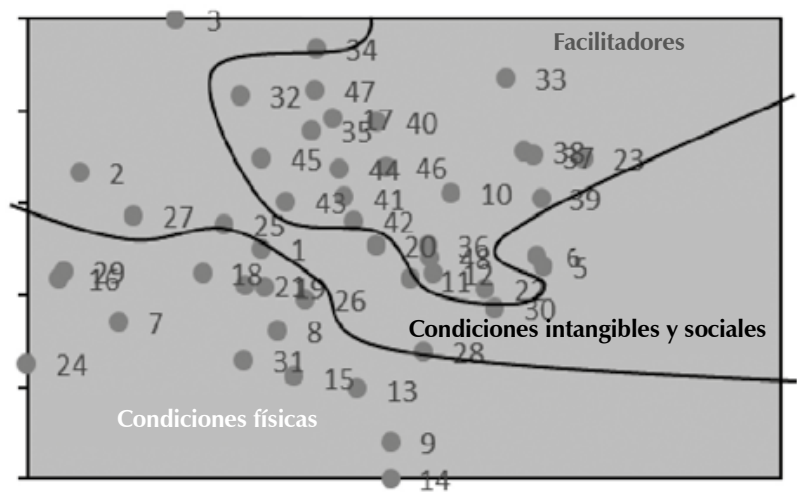

dos ciudades). Finalmente, la dimensión "Condiciones espaciales y sociales" dio lugar también a tres regiones, que se evidenciaron en todas las ciudades, y que se refieren a: 1) las condiciones físicas, 2) los facilitadores del caminar y 3) las condiciones sociales y a las que se consideraron intangibles. Dentro de estas últimas se agrupan los ítems que valoran la limpieza del lugar, el clima, la iluminación, la estética del barrio, la posibilidad de encuentros sociales, y la continuidad de las aceras, e inhibidores como la congestión
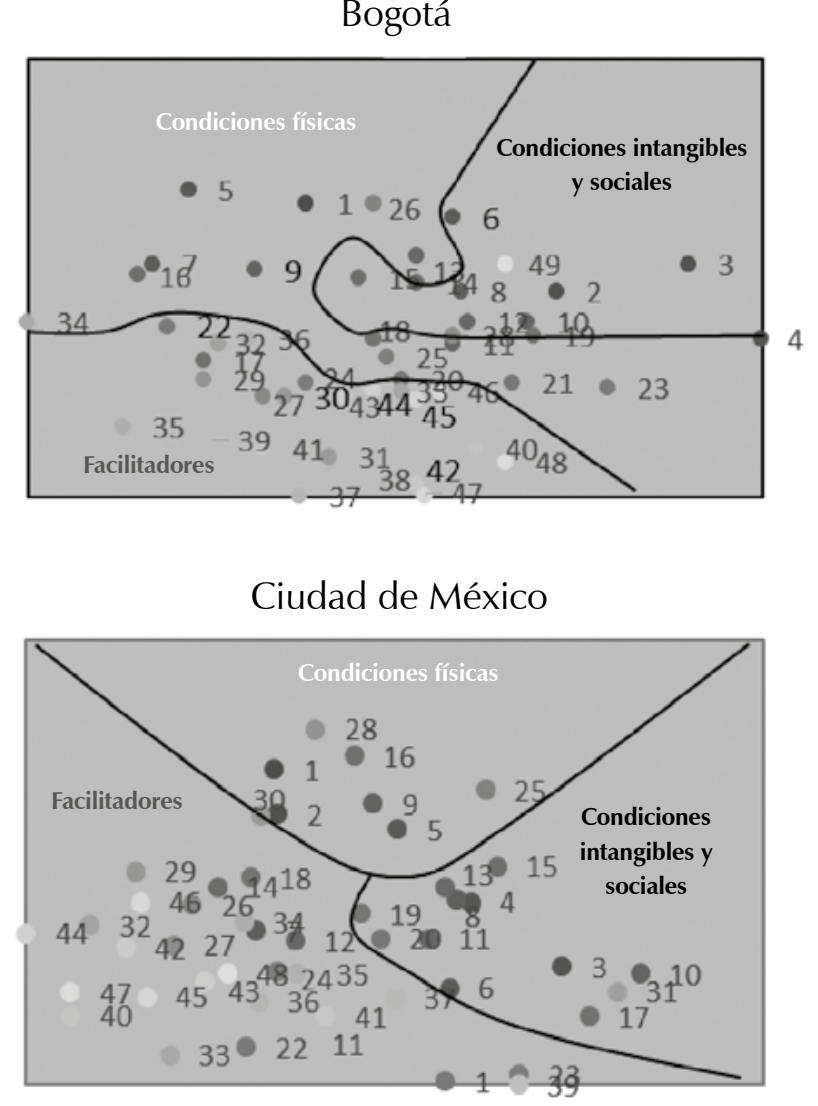

Montevideo

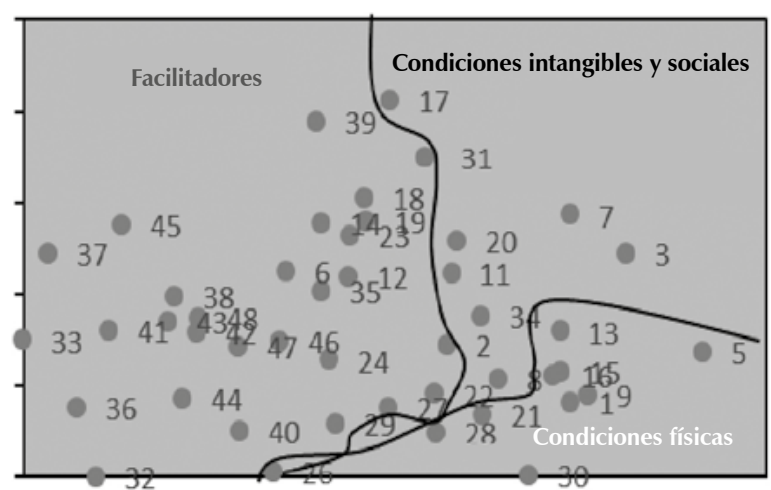

Tunja

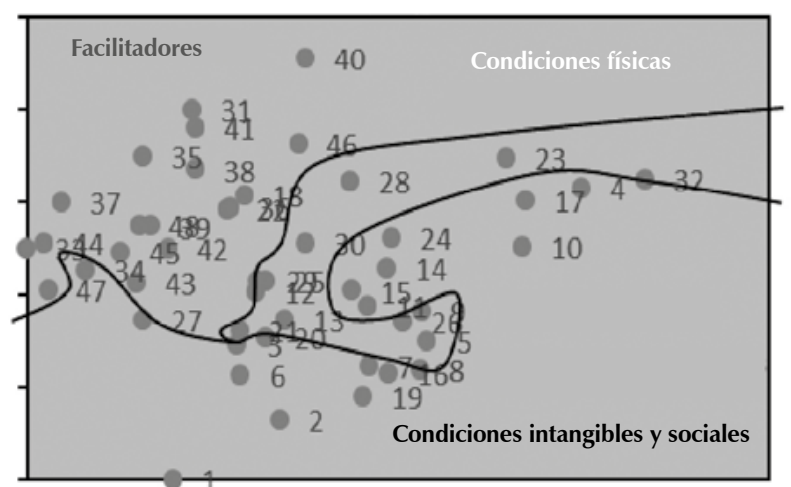


vehicular, la calidad del aire, la lluvia, el ruido de las calles y la ocupación indebida del espacio público, como se muestra en la figura 3.

\section{Sobre la seguridad de las mujeres que caminan}

El análisis de la pregunta 49 evidenció marcadas diferencias entre varias ciudades respecto a la valoración que hicieron las mujeres participantes del estudio sobre la caminabilidad de la ciudad en la condición de mujer. Las más notorias se observan entre Guadalajara (México) y Tunja (Colombia), por cuanto la primera aparece como muy poco caminable para pasear sola sin ser acosada, y la segunda, como más caminable. Pero, por lo general, las participantes de todas las ciudades analizadas consideran que las ciudades se ubican entre muy poco caminables y medianamente caminables, sin sentirse acosadas cuando caminan por sus calles, teniendo en cuenta que la escala iba de 1 a 7 grados, como se muestra en la figura 4.

\section{Análisis de la caminabilidad general}

Al comparar la valoración de la caminabilidad general (ítem 50), se construyó la figura 5, a partir de los promedios obtenidos de la calificación de la habitabilidad general de las ciudades de forma ascendente, y donde se observa que Arequipa es la ciudad evaluada con el menor promedio, y Talca, la mejor evaluada, aunque con pequeñas diferencias entre ellas, lo cual indica que las ciudades, en general, son evaluadas como medianamente caminables, teniendo en cuenta que la escala iba de 1 a 7.

\section{Resultados de las entrevistas}

Enseguida se presenta una síntesis de las entrevistas, las que estuvieron dirigidas a explorar en profundidad: los propósitos al caminar; lugares y las condiciones socioespaciales; la experiencia y la corporalidad, y finalmente, caminar como experiencia fenomenológica.

En cuanto a Propósitos al caminar, estos se pueden diferenciar entre los que tienen que ver con motivos de orden instrumental —como dirigirse al trabajo, a la escuela o a hacer comprasy aquellos que se pueden definir como lúdicos o recreativos -tales como distraerse, pasear o socializar, y por motivos de salud-. Dichas metas, si bien establecidas como predominantes, también pueden estar interrelacionadas, como es el caso de caminar al trabajo con audífonos para escuchar música o en la dinámica cotidiana hacer pequeños desvíos hacia centros comerciales o a ver amigos. Caminar también puede estar clasificado de acuerdo con su temporalidad: en días laborales de la semana, para dirigirse al trabajo, de compras o a la escuela, y los fines de semana, para pasear. El mismo principio opera al dividir el día en segmentos: la mañana, con sus
¿Qué tan caminable es su ciudad para pasear sola por la ciudad sin ser acosada?

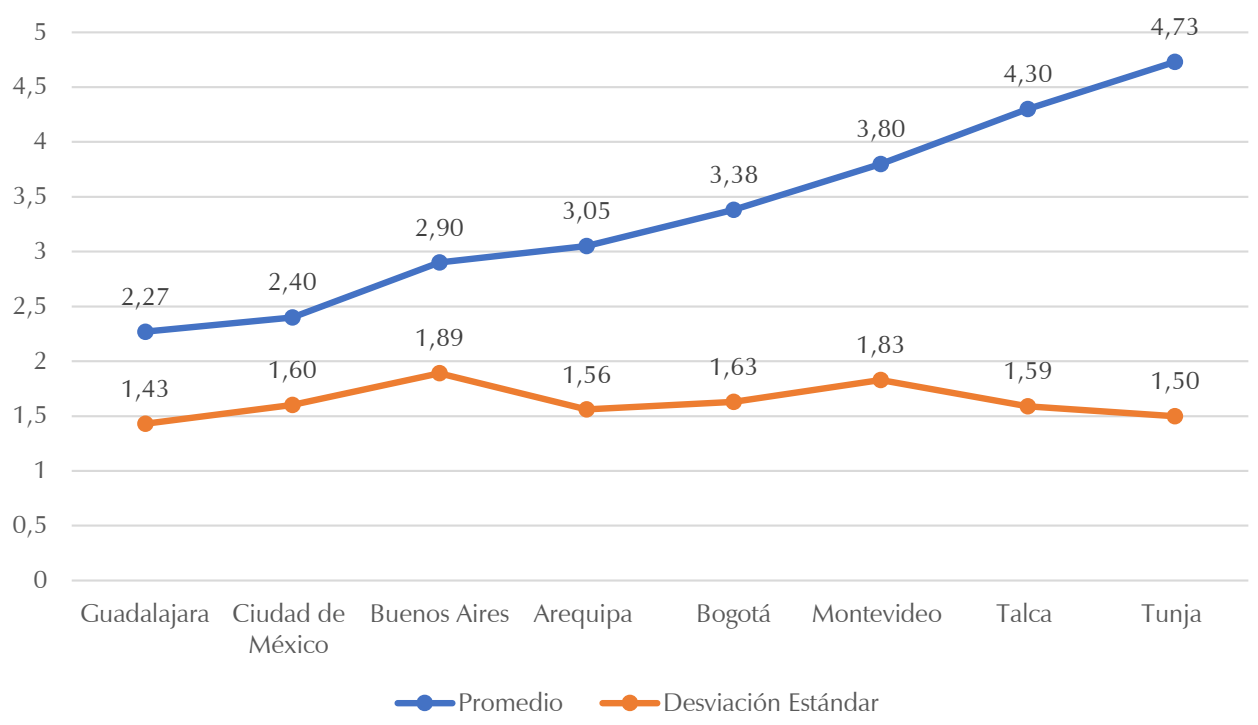

(4) Figura 4. Comparación entre ciudades respecto de qué tan caminable es la ciudad para las mujeres sin sentirse acosadas. Fuente: elaboración propia (2020). CC BY-NC

¿Qué tan caminable es su ciudad?

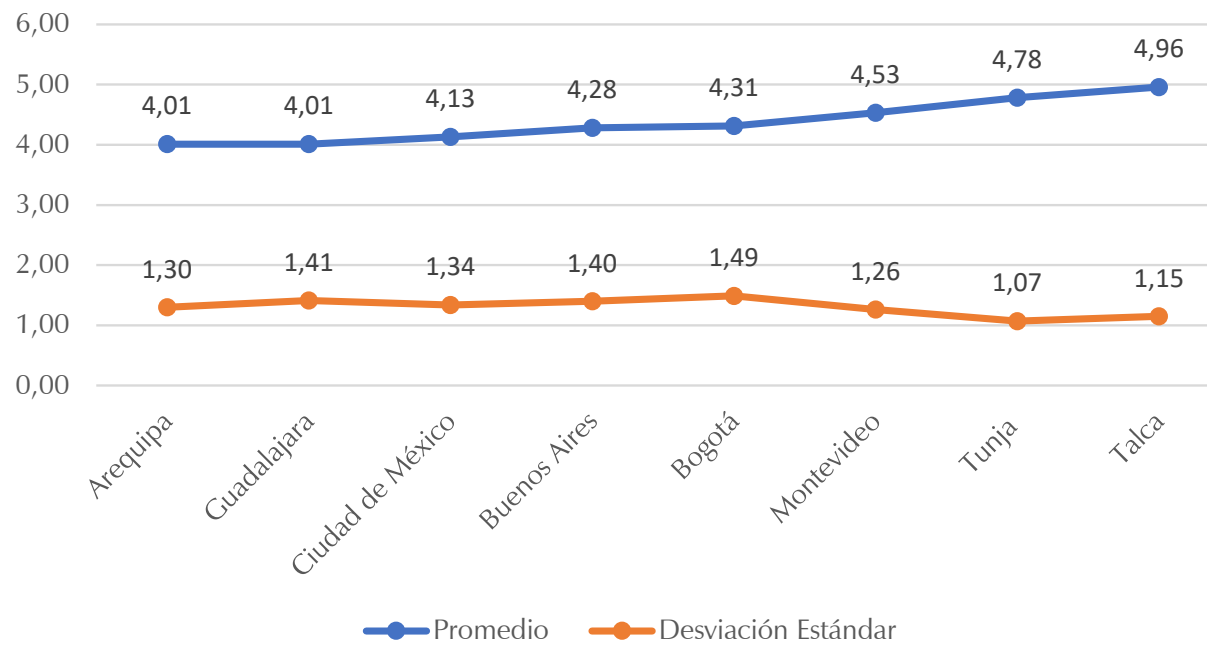

traslados obligados, y la tarde o la noche, en las que son las decisiones y las preferencias individuales las que mueven a las personas. También se menciona como propósito de caminar, el hecho de salir a pasear a las mascotas, o bien, recorrer la ciudad con la intención expresa de tomar fotografías, lo cual, a su vez, revela que hay un tipo de actividades intersticiales, que generan desplazamientos, principalmente locales (como es el caso de las mascotas), y otras a una escala metropolitana, como sería la deriva visual de los elementos paisajistas de carácter patrimonial que hacen parte del ambiente construido que atraen la mirada del transeúnte. Es claro que caminar se encuentra estructurado por lo cotidiano; es decir, por las rutinas que se llevan a cabo en la ciudad como parte de la vida cotidiana. Ir a la escuela o al trabajo, salir de compras y visitar amigos o parientes requieren un desplazamiento que no es azaroso: cuenta con tiempos y lugares
(4) Figura 5. Valoración genera sobre la caminabilidad de las ciudades. Fuente: elaboración propia (2020). CC BY-NC. 
que son parte de los ritmos y la localización de estas actividades; de ahí que los propósitos de caminar no sean de ninguna manera ajenos a la forma social de lo urbano.

Respecto a Lugares y condiciones socio-espaciales, la idea de ruta prevalece sobre los lugares. Implica esto que a la hora de caminar, el rumbo por seguir importa, en general, más que la referencia al lugar o los lugares a los que se llega. El tránsito, al mismo tiempo, no es absolutamente libre, sino que se ciñe a un camino transitado con regularidad, donde la experiencia y el conocimiento previos son importantes en la elección del caminante, ya que proporcionan seguridad y reconocimiento de los diversos lugares del recorrido. A pesar de lo anterior, para algunas personas recorrer todos los días la misma ruta no es sinónimo de seguridad, sino, más bien, de monotonía, de tal forma que prefieren cambiar el camino por el que andarán. No hay, entonces, una manera única de trazar los desplazamientos por la ciudad: la heterogeneidad de estrategias de movimiento es parte del caminar urbano. Cabe también apuntar que para varios entrevistados tener compañía al momento de salir a caminar es parte de la ruta misma; hay trayectos que se efectúan con determinadas personas, y no con otras. Esto recuerda lo planteado por Lee e Ingold (2006) en relación con la sociabilidad y la ruta al caminar. Si bien es cierto que los desplazamientos se cuentan como un tránsito por diferentes rutas, también lo es que son las áreas urbanas centrales las que concentran las preferencias al momento de señalar los lugares transitados. En Arequipa se menciona la central Plaza de Armas. En Guadalajara se mencionaron diferentes zonas, centrales o periféricas, pero fácilmente reconocibles, como Zapopan, Tlaquepaque y el mismo centro de la ciudad. En Montevideo se señalan Las Ramblas, una extensa avenida que bordea al Río de la Plata. En Bogotá, la central Carrera Séptima es un punto de referencia, lo mismo que la intensa y juvenil Plaza del Chorro de Quevedo. Al hablar los entrevistados sobre los lugares por los que transitan — sean parques, plazas o calles-, en muchos casos aluden a puntos de referencia urbanos ubicados en áreas urbanas céntricas, o bien, que cuentan con un alto valor histórico y patrimonial. Esto es interesante, por cuanto muestra que caminar toma un carácter de actividad especial al efectuarse en áreas que forman parte de la imagen dominante de la ciudad. En algunos casos, las áreas por las que se camina remiten a valores históricos, patrimoniales naturales o culturales; un elemento en juego en la elección del lugar por el que se camina es, entonces, el tipo de experiencia a la que se busca acceder, sin olvidar, claro, que la sorpresa siempre está ahí. Otro elemento, en muchos casos crucial, y que define la ruta por seguir, es el de la seguridad. Mayoritariamente para las mujeres, la elección de la ruta está orientada a evitar situaciones de acoso callejero, robo y violencia. Tan aguda percepción se vuelve un obstáculo para la movilidad peatonal, en términos de buscar medios de transporte pensados como más seguros, al igual que de la misma experiencia de estar en la calle, dado que, en situación de percepción de vulnerabilidad, las personas se centran en el cuidado de sí, y no están atentas a otros estímulos ambientales.

Al hablar de los traslados cotidianos, algo que se señala con regularidad es la molestia frente a obstáculos materiales en las calles y las aceras, y que podrían tratarse de autos mal estacionados, postes de servicios telefónicos, basura o mercancía en la vía pública. Conspira también contra el movimiento fluido del peatón la escasa educación vial por parte de los conductores de autos, lo cual hace que atravesar la calle se haga en condiciones de riesgo, y ello obligue al peatón a desplazarse no por la vía preferida, sino por la que queda libre entre los objetos, o bien, en condiciones de prisa y vigilancia al movimiento de los autos.

Estas dificultades son aún más importantes cuando las personas tienen que movilizarse en silla de ruedas o empleando muletas. La saturación vinculada con la movilidad es mencionada en relación con las aglomeraciones en el transporte público; de ahí que se prefiera, cuando es posible, caminar en vez de trasladarse por otros medios. Todos los indicadores que remiten a una atmósfera de saturación revelan, a su manera, la insatisfacción con las condiciones en que se lleva a cabo la movilidad urbana. Los peatones encuentran que el desplazamiento es difícil, mientras que los usuarios del transporte buscan evitar su uso cuando es posible.

Sobre Experiencia y corporalidad, la ciudad sensible evocada por los entrevistados se compone de una gama amplísima de referencias. Se informa del olor del pan recién horneado, de ver y oler flores y árboles, de encontrar placer en el aroma a césped húmedo y en sentir y reconocer la textura del piso por el que se camina. Los olores que se desprenden de las cañerías y las fábricas son repelentes. Al caminar se reconocen barrios a partir de su olor y sus sonidos. Hay también quien al caminar escucha la tranquilidad de la noche, y otros disfrutan el sonido de los pasos de quienes caminan en la madrugada, o bien, van tocando la textura de las paredes de las casas al desplazarse. La lluvia cae en la piel y produce, fugazmente, otra sensibilidad en la ciudad. Se observan las diferencias entre las personas en las calles a partir de su vestimenta y del cuidado que ponen en su apariencia. Al caminar se produce un estado de ánimo: la lentitud produce relajación, y la prisa es rápida y tensa; más aún, la sensación de apuro produce que se camine no sobre el espacio, sino en la superficie del tiempo, con la consciencia de que hay que 
desplazarse lo más rápido posible. En lugares obscuros se agudizan los cinco sentidos, se está alerta ante la inseguridad. La conversación de los otros peatones es objeto de escucha, mirarse a sí mismos en vidrios y aparadores es un gusto fugaz. Al momento de recrear dimensiones sensoriales experimentadas al caminar, el centro de la ciudad es nombrado recurrentemente. Caminar aquí adquiere una riqueza particular que no se encuentra en otros espacios. Hay un aprecio del pasado, de la arquitectura, una pedagogía de lo urbano en el reconocimiento de transformaciones arquitectónicas. Los testimonios vertidos apuntan a pensar en el pasado casi como un objeto que tuviera sus propios rasgos sensibles, y que fuera capaz de ser encontrado en el centro de la ciudad. Por encima de sus amplios usos comerciales y patrimoniales, habría, entonces, la búsqueda de un pasado que se puede recuperar sensorialmente al caminar por sus calles y sus plazas. El énfasis en las diversas e interminables sensaciones sensoriales muestra a la ciudad como un espectáculo al que se accede caminando. De tal espectáculo no está exento lo desagradable ni lo inesperado, aunque cabe destacar que cuando se pregunta sobre qué es lo que más llama la atención al caminar y qué aspecto sensorial se recuerda particularmente, son los elementos placenteros los que aparecen con intensidad. Caminar, desde esta óptica, tiene que ver con el arte de apreciar lo pequeño y unirlo en la distancia a través de los pasos. La percepción sensorial que tiene aquel que camina por calles y barrios de la ciudad tiene la característica de traducir la ciudad a una escala humana, donde lo que ocurre alrededor puede ser apreciado con amplitud.

Indudablemente, la apropiación sensorial de la ciudad ocurre al caminar. Se reconocen calles y barrios por olores y sonidos, por aquello que se mira. Todo ello apunta hacia un orden sensorial urbano, en el que distintos rasgos sensoriales se encuentran ubicados en áreas particulares de la ciudad; así, habrá zonas más verdes que otras, más ruidosas y tranquilas, con más olor a basura, o con menos. La localización de estos rasgos sensibles se conjuga con elementos que son parte de diferenciaciones socioeconómicas de la ciudad, y se generan así estos mapas, inicialmente sensibles, que pueden devenir en cartografías sensibles y afectivas, donde las valoraciones sensibles remiten a estados afectivos, y viceversa.

En Experiencia fenomenológica, el interés del acercamiento a la experiencia fenomenológica estriba en abordar qué pasa por la mente de los caminantes por la ciudad en sus traslados cotidianos; es decir, sobre qué reflexionan, qué tipo de pensamiento produce la combinación de movimiento, corporalidad y presente personal. Cabe apuntar que son múltiples los matices metodológicos del abordaje fenomenológico, y señalamos solamente que partimos de las evocaciones y el discurso de los entrevistados en relación con caminar en la ciudad. Al respecto, el contraste entre el pasado y el presente (cambios en la morfología de la ciudad, en las personas, en las costumbres, e incluso, en el mismo transeúnte) es algo que se reflexiona al caminar. En este sentido, ser peatón es un ejercicio en la práctica del sí mismo, una actividad que hace emerger y da forma a puntos de referencia biográficos. La familia es un tema presente en las reflexiones: alguien se pregunta si la familia ya llegó bien a casa, y otra persona recuerda que caminaba recurrentemente con su padre. La nostalgia es también un recurso para relacionarse con las transformaciones; de ahí que se diga que antes todo era más tranquilo, y no había situaciones como las que hay ahora (en referencia a la inseguridad). Así, los relatos de caminar muestran también que caminar por la ciudad puede dar lugar a la reflexión y al encuentro consigo mismo. Moverse por la ciudad en soledad permite abordar situaciones personales, conocerse a sí mismo como intérprete de situaciones y rasgos en la ciudad. Algunos participantes imaginan historias a partir de lo que observan y lo que fantasean en sus recorridos. También hay quien reflexiona desde las imágenes con su cámara fotográfica como forma de apropiarse de los lugares de la ciudad.

\section{Discusión y conclusiones}

El estudio permite concluir que caminar tiene importantes implicaciones en la vida de las personas, y en general, en la calidad de vida urbana, por cuanto contribuye a mejorar sus condiciones de salud, facilitar su movilidad y apropiarse de la ciudad. Además, caminar en los espacios públicos urbanos contribuye a los encuentros culturales, sociales, recreativos y de consumo, con lo que se hace un aporte significativo a la calidad de vida urbana y a la economía de las ciudades. Desde lo fenomenológico, contribuye a la memoria y la afectividad del individuo, a aprender del paisaje y a conformar democráticamente la ciudad.

En respuesta a la pregunta de investigación, se identificaron tres propósitos principales por los que las personas caminan: 1) para movilizarse hacia o desde el lugar de estudio o de trabajo hacia su hogar y para adelantar diligencias tales como pagar servicios, asistir al colegio de los hijos, hacer compras, realizar gestiones ante bancos, etc.; 2) mejorar sus condiciones de salud y prevenir enfermedades; 3) por recreación y ocio. Los participantes, así mismo, diferencian el acto de caminar a partir de tres escenarios: 1) el centro comercial, 2) los espacios públicos construidos o naturales y 3) el barrio, aunque con algunas excepciones para unas ciudades respecto del barrio. E identifican tres condiciones sociales y espaciales facilitadoras o inhibidoras de caminar: 1) los oferentes del espacio, 2) las 
situaciones que identificamos como intangibles y 3) las sociales.

Se destaca que los ítems con medias más altas son los relativos a que la ciudad es caminable en los centros comerciales -muy seguramente, por la sensación de seguridad que representa-; para encontrarse con los amigos; y para pasear al perro, que juega, en este sentido, un papel importante en la salud de las personas al comprometerlas con el caminar, además de actuar como oferente de encuentros sociales. Los ítems valorados menos favorablemente son los que hacen referencia a la seguridad, al acoso que experimentan las mujeres y a la posibilidad de encontrar un policía; pero, igualmente, con la insuficiencia de mobiliario urbano como baños públicos y la existencia de dispensadores de agua. Igualmente, sobresale en los datos que la desviación estándar de los ítems en relación con sus medias es baja entre las ciudades, lo cual indica que hay bastante grado de acuerdo en la valoración que se hace de caminar en todas las ciudades de la región.

El estudio hace un aporte a la arquitectura y al urbanismo en general, en la medida en que se identifican las distintas motivaciones por las que se camina en distintos escenarios y las condiciones espaciales y sociales que dificultan o contribuyen a la caminabilidad de las ciudades, con lo cual se dan pautas a los diseñadores y los gestores urbanos para lograr este objetivo haciendo que las ciudades cuenten con mayor y mejor infraestructura. Por ejemplo, introduciendo un mayor número de kilómetros caminables; elementos estéticos, como obras de arte y paisajísticos; proveyendo los espacios públicos de mobiliario urbano, como bancas, baños públicos, luminarias, demarcación y señales para el cruce seguro de las avenidas para los peatones, etc.

Un aspecto fundamental que podría incentivar a caminar, con la perspectiva de la planeación derivada del presente estudio, es la recuperación del barrio como unidad espacial urbana delimitándolo espacialmente y conectando sus equipamientos mediante vías peatonales, o creando manzanas o bloques cerrados para vehículos, a la manera como lo vienen haciendo en Barcelona y Nueva York.

Pero no es suficiente con la infraestructura física: es esencial que las instituciones gubernamentales garanticen la seguridad de los espacios públicos, y que así sean percibidos por los ciudadanos. Es necesario que desde las instituciones educativas y las encargadas de promover la salud y el deporte, se promueva caminar mediante el diseño de políticas públicas, para lo cual resulta esencial crear programas que promuevan ir a la escuela o al trabajo caminando, y para ello, a la vez, será necesario su diseño desde las instituciones de gobierno, así como crear indicadores que permitan hacer su seguimiento de estas.
Dadas las nuevas condiciones de desplazamiento y emplazamiento de las ciudades en crecimiento, se plantea el reto de explorar con diferentes metodologías el acto de caminar. La utilización de un cuestionario y de entrevistas semiestructuradas permitió identificar las motivaciones, las limitaciones propias de caminar y los lugares por donde se camina, a partir de las valoraciones de los participantes; sin embargo, para que la información obtenida sea más válida y confiable, en términos de su generalización, habría que, en primer lugar, replicar el estudio y, en lo posible, tomar muestras más representativas y estratificadas a partir de comunas o localidades, género, grupos de edad y zonas rurales y urbanas, entre otras variables para tener en cuenta. En segundo lugar, es indispensable complementar esta información con indicadores objetivos: por ejemplo, valiéndose del Sistema de Información Geográfica y otros que deberían crearse. Cabe suponer que a mayor diversidad geográfica, territorial y climatológica, y a mayor cantidad de áreas verdes, etc. de una ciudad, mayores son la diversidad y las formas de caminar, asuntos que deberían investigarse en mayor detalle en futuras investigaciones.

\section{Contribuciones y agradecimientos}

El estudio contó con la colaboración de: Felipe Coronado, Alexandra Prado y Camilo Moreno, en la sistematización de la información de campo, y quienes hicieron parte del equipo de trabajo del proyecto institucional El Caminar como Experiencia de Aprendizaje en la Ciudad, de la Universidad Pedagógica Nacional, de Bogotá, Colombia. Igualmente, se agradece a Yareli Lisbeth Palacios Gómez su apoyo para sistematizar la información de las entrevistas a profundidad de las ocho ciudades. De igual manera, nuestros agradecimientos se extienden al grupo de investigación Espacios Públicos-UNSA (Arequipa, Perú): Erika Guerra Santander, Smilzinia Huerta Tapia y Katherine Ancco Encina.

La contribución de los autores consistió en la recolección y en el análisis de datos de cada una de las ciudades participantes del estudio:

- Bogotá: Pablo Páramo y Andrea Burbano. Autores que, a su vez, coordinaron la investigación y la escritura del artículo.

- Tunja: Guillermo Rosas.

- Arequipa: Edgar García Anco y Edward Silvestre Pari Portillo.

- Guadalajara: Bernardo Jiménez-Domínguez y Rosa Margarita López Aguilar.

- Ciudad de México: Miguel Ángel Aguilar. Su contribución se orientó, además, al análisis de los datos provenientes de las entrevistas de todas las ciudades.

- Talca: Emilio Moyano Díaz.

- Montevideo: Eduardo Viera.

- Buenos Aires: Ángel Manuel Elgier. 


\section{Referencias}

AAA. (2018). Foundation for Traffic Security. 2018 Traffic Safety Culture Index. https://aaafoundation.org/2018-trafficsafety-culture-index/

Amar, R. (2001). HUDAP. Hebrew University Data Analysis Package (software). Hebrew University Computation Centre, Jerusalem.

Aguilar, M. A. (2016). El caminar urbano y la sociabilidad. Trazos desde la ciudad de México. Revista Alteridades. 26(52). 23-33. http://www.scielo.org.mx/scielo.php?script=sci arttext\&pid=S0188-70172016000200023\&lng $=\mathrm{es} \&$ tlng $=\mathrm{es}$

Aguilar, M. A. (2018). Memoria y afecto en el caminar urbano. En E. Calderón, y A. Zirión (Coords.), Cultura y afectividad. Aproximaciones antropológicas y filosóficas al estudio de las emociones. Ediciones del Lirio/Universidad Autónoma Metropolitana.

Bassett, D. R. Jr, Pucher, J., Buehler, R., Thompson, D. L., y Crouter, S. E. (2008). Walking, cycling, and obesity rates in Europe, North America, and Australia. Journal of Physical Activity \& Health, 5(6), 795-814. https://doi.org/10.1123/jpah.5.6.795

Borg, I., Groenen, P. J. F. (2005). Modern multidimensional scaling. Springer.

Borg, I. y Shye, S. (2005). Facet Theory: Form and Content. Sage.

Burbano, A. (2014). El espacio público urbano situado en la ciudad latinoamericana contemporánea: una aproximación a su estudio desde la perspectiva del género. En E. Licona (Ed.), Espacio y Espacio Público contribuciones para su estudio. Benemérita Universidad de Puebla (BUAP).

Burbano, A. (2016a). Modelo territorial para el estudio del espacio público urbano con perspectiva de género. El caso de una ciudad de Latinoamérica contemporánea (1991-2012) (Tesis doctoral). Universidad de Caldas, Manizales (Colombia).

Burbano, A. (2016b). Espacio y género. En: E. Licona y V. Cabrera (Eds.). Para pensar el territorio. Elementos epistémicos y teóricos. Benemérita Universidad de Puebla (BUAP).

CEPAL. (2011). Experiencias Internacionales en campañas integrales y efectivas de seguridad vial. CEPAL.

Conejera, M., Donoso, D., Moyano, E., y Peña, J. (2003). Comunicación persuasiva y cambio de actitudes hacia la seguridad de tránsito en peatones. Revista Latinoamericana de Psicología, 35(1), 77-90.

https://dialnet.unirioja.es/servlet/ articulo?codigo $=2834616$

D’Alessandro, D., Appolloni, L., y Capasso, L. (2016). How walkable is the city? Application of the Walking Suitability Index of the Territory (T-WSI) to the city of Rieti (Lazio Region, Central Italy). Epidemiología e prevenzione, 40(3-4), 237 https://doi.org/10.1177/0265813515625641

De Certeau, M. (1984). Walking in the city. The University of California Press.

Falú, A. (2009). Violencias y discriminaciones en las ciudades. En A. Falú (Ed.). Mujeres en la ciudad. De violencias y derechos. Santiago de Chile: Red Mujer y Hábitat de América Latina Ediciones SUR. http://americalatinagenera.org/newsite/ images/cdr-documents/publicaciones/libro mujeres_en_la_ciudad.pdf

Forjuoh, S. N., Ory, M. G., Won, J., Towne, S D., Wang, S. y Lee, C. (2017). Determinants of walking among middle-aged and older overweight and obese adults: Sociodemographic, health, and built environmental factors. Journal of Obesity, J Obes. Article ID 9565430, 11.

https://doi.org/10.1155/2017/9565430

Fundación Gonzalo Rodríguez. (2018). Programa de Niños Latinoamericanos Seguros. https://www.gonzalorodriguez.org/ uploads/memoria/089685e248a673fa891301157d44a5f31e2c5cfe.pdf

Fundación MAPFRE. (2016).

https://www.mapfre.com/corporativo-es/ acerca-mapfre/fundacion-mapfre/

Gutiérrez-López, J. A., Caballero-Pérez, Y. B. y Escamilla-Triana, R. A. (2019). Índice de caminabilidad para la ciudad de Bogotá. Revista de Arquitectura (Bogotá), 21(1), 8-20. http://dx.doi.org/10.14718/RevArq.2019.21.1.1884

Hackett P. M. W. (2014). Facet Theory and the Mapping Sentence: Evolving Philosophy, Use and Application. Palgrave.

Hackett P. M. (2016). Facet theory and the map ping sentence as hermeneutically consistent structured meta-ontology and structured meta-mereology. Front. Psychol. 7:471.

Haines A., McMichael A. J., Smith K. R, Roberts I, Woodcock, J. y Markandya A, (2009). Public health benefits and strategies to reduce greenhouse-gas emissions: overview and implications for policy makers. Lancet. 374(9707), 2104-2114. [PubMed] https://doi.org/10.1016/S0140-6736(09)61716-5

Hollenstein, D., y Bleischa, S. (2016). Walkability for different urban granularities. ISPRS-International Archives of the Photogrammetry, Remote Sensing and Spatial Information Sciences, 41, 703-708.

https://doi.org/10.5194/isprs-archivesXLI-B2-703-2016

Jacobs, J. (1961). The death and life of great American cities. Vintage Books.

Keller, C. y Cantue, A. (2008). Camina por Salud: Walking in Mexican-American women. Applied Nursing Research, 21(2), 110-113. https://doi.org/10.1016/j.apnr.2006.12.003

Kerr, J., Emond, J. A., Badland, H., Reis, R., Sarmiento, O., Carlson, J., Sallis, J. F., Cerin, E. Cain, K., Conway, T., Schofield, G., Macfarlane, D. J., Christiansen, L. B., Van Dyck, D., Davey, R., Aguinaga-Ontoso, I., Salvo, D., Sugiyama, T., Owen, N., Mitáš, J., Natarajan, L. (2016). Perceived neighborhood environmental attributes associated with walking and cycling for transport among adult residents of 17 cities in 12 countries: the IPEN study. Environmental Health Perspectives (124), 290-298. http://dx.doi.org/10.1289/ehp.1409466

Le Breton, D. (2007). El sabor del mundo. Una antropología de los sentidos. Nueva Visión.

Lee, J. y Ingold, T. (2006). Fieldwork on foot: Perceiving, routing, socializing. En P. Collins y S. Coleman (Eds.), Locating the Field. Space, place and context in anthropology (pp. 67-86). Berg.

Lefebvre, H. (1969). El derecho a la ciudad. Península [edición original: (1968) Le Droit à la ville, Paris: Anthropos].

Lindón, A. (2006). Territorialidad y género: una aproximación desde la subjetividad espacial. En Pensar y habitar la ciudad: Afectividad, memoria y significado en el espacio contemporáneo (pp. 13-33). UAM Iztapalapa y Editorial Anthropos.

https://dialnet.unirioja.es/servlet/ articulo?codigo $=1425064$

Low, S. (2005). Transformaciones del espacio público en la ciudad latinoamericana: cambios espaciales y prácticas sociales. Bifurcaciones, 5, 1-14.
Moyano Díaz, E. (2002). Theory of planned behavior and pedestrians' intentions to violate traffic regulations. Transportation Research Part F: Traffic Psychology and Behaviour, 5(3), 169-175 https://doi.org/10.1590/S1413-294X1997000200008.

Moyano Díaz, E. (1999). Psicología Social y Seguridad de Tránsito. Universidad de Santiago de Chile.

Oldemburg, R. (2013). Prólogo al libro Sociolugares. En P. Páramo, Sociolugares. Ediciones Universidad Piloto de Colombia.

OMS-OPS. (2016). La seguridad vial en la región de las Américas.

www.who.int/violence injury prevention/ road_safety_status/2015/Road_Safety PAHO__Spanish.pdf

Ortiz, A. (2007). Hacia una ciudad no sexista. Algunas reflexiones a partir de la geografía humana feminista para la planeación de espacio urbano. Revista Territorios. 16(17) $11-28$

https://revistas.urosario.edu.co/index.php/ territorios/article/view/838

Páramo, P. (2011). Sociolugares. Ediciones Universidad Piloto de Colombia.

Páramo, P. y Burbano, A. (2019). La caminabilidad en Bogotá: propósitos y condiciones socio-espaciales que facilitan y limitan esta experiencia. Revista de Arquitectura (Bogotá), 21(2). http://dx.doi.org/10.14718/RevArq.2019.21.2.2642

Páramo, P., Burbano, A., Jiménez-Domínguez, B., Barrios, V., Pasquali, C., Vivas, F., Moros, O., Alzate, M., Jaramillo, J. C. y Moyano, E. (2018). La habitabilidad del espacio público en las ciudades de América Latina. Avances en Psicología Latinoamericana, 36(2), 345-362. http://dx.doi.org/10.12804/revistas.urosario. edu.co/apl/a.4874

Poe, E. A. (1972 [1840]). El hombre de la multitud. En Julio Cortázar (selecc. y trad.), Cuentos I. Alianza.

http://files.el-magnifico-allanpoe.webnode. com.co/200000042-1635d172f5/Cuentoscompletos-de-Allan-Poe-Traduccion-JulioCortazar.pdf

Sennett, R. (1977). El declive del hombre público. Península.

Speck, J. (2012). Walkable city: How downtown can save America. One Step at a Time. North Point Press.

Talavera-García, R., y Soria-Lara, J. A. (2015). Q-PLOS, developing an alternative walking index. A method based on urban design quality. Cities. 45, 7-17 http://dx.doi.org/10.1016/j.cities.2015.03.003

Torres, A. (2019). La experiencia de aprendizaje al caminar las ciudades latinoamericanas vista desde el análisis de sus políticas públicas, documentación técnica y encuentros académicos y sociales. (Tesis de Maestría). Universidad Pedagógica Nacional, Bogotá.

Tribby, C. P., Miller, H. J., Brown, B. B., Werner, C. M., y Smith, K. R. (2016). Assessing built environment walkability using activity-space summary measures. Journal of transport and land use, 9(1), 187. http://dx.doi.org/10.5198/jtlu.2015.625

Walk21 - Leading the Walking Movement. (2018). Walk21- XIX: Bogotá, Colombia. Walkable City, 15-19 de octubre de 2018. https://www.walk21.com/bogota 
A Portada: Catedral de Notre Dame de Paris durante los trabajos de reparación en octubre de 2020 Fotografía: Arquitecto Andrés Avila Gómez (octubre, 2020)
(A) Orientación editorial

\section{Enfoque y alcance}

La Revista de Arquitectura (Bogotá) ( (ISSN 1657-0308 Impresa y E-ISSN 2357-626X en línea) es una publicación científica seriada de acceso abierto, arbitrada mediante revisión por pares (doble ciego) e indexada, en donde se publican resultados de investigación originales e inéditos.

Está dirigida a la comunidad académica y profesional de las áreas afines a la disciplina. Es editada por la Facultad de Diseño y el Centro de Investigaciones (CIFAR) de la Universidad Católica de Colombia en Bogotá (Colombia).

La principal área científica a la que se adscribe la Revista de Arquitectura (Bogotá) según la OCDE es:

Gran área: 6. Humanidades

Área: 6.D. Arte

Disciplina: 6D07. Arquitectura y Urbanismo

También se publican artículos de las disciplinas como 2A02, Ingeniería arquitectónica; 5G03, Estudios urbanos (planificación y desarrollo); 6D07, Diseño.

Los objetivos de la Revista de Arquitectura (Bogotá) son:

- Promover la divulgación y difusión del conocimiento generado a nivel local, nacional e internacional

- Conformar un espacio para la construcción de comunidades académicas y la discusión en torno a las secciones definidas.

- Fomentar la diversidad institucional y geográfica de los autores que participan en la publicación.

- Potenciar la discusión de experiencias e intercambios científicos entre investigadores y profesionales.

- Contribuir a la visión integral de la arquitectura, por medio de la concurrencia y articulación de las secciones mediante la publicación de artículos de calidad.

- Publicar artículos originales e inéditos que han pasado por revisión de pares, para asegurar que se cumplen las normas éticas, de calidad, validez científica, editorial e investigativa.

- Fomentar la divulgación de las investigaciones y actividades desarrolladas en la Universidad Católica de Colombia.
Palabras clave de la Revista de Arquitectura (Bogotá): arquitectura, diseño, educación arquitectónica, proyecto y construcción, urbanismo.

Idiomas de publicación: español, inglés, portugués y francés.

Título abreviado: Rev. Arquit.

Titulo corto: RevArq

\section{Políticas de sección}

La revista se estructura en tres secciones correspondientes a las líneas de investigación activas y aprobadas por la institución, y dos complementarias, que presentan dinámicas propias de la Facultad de Diseño y las publicaciones relacionadas con la disciplina.

Cultura y espacio urbano. En esta sección se publican los artículos que se refieren a fenómenos sociales en relación con el espacio urbano, atendiendo aspectos de la historia, el patrimonio cultural y físico, y la estructura formal de las ciudades y el territorio.

Proyecto arquitectónico y urbano. En esta sección se presentan artículos sobre el concepto de proyecto entendido como elemento que define y orienta las condiciones proyectuales que devienen en los hechos arquitectónicos o urbanos, y la forma como estos se convierten en un proceso de investigación y nuevo de conocimiento. También se presentan proyectos que sean resultados de investigación, los cuales se validan por medio de la ejecución y transformación en obra construida del proceso investigativo. También se contempla la publicación de investigaciones relacionadas con la pedagogía y didáctica de la arquitectura, el urbanismo y el diseño.

Tecnología, medioambiente y sostenibilidad. En esta sección se presentan artículos acerca de sistemas estructurales, materiales y procesos constructivos, medioambiente y gestión, relacionados con los entornos social-cultural, ecológico y económico.

Desde la Facultad. En esta sección se publican artículos generados en la Facultad de Diseño, relacionados con las actividades de docencia, extensión, formación en investigación o internacionalización, las cuales son reflejo de la dinámica y de las actividades realizadas por docentes, estudiantes y egresados; esta sección no puede superar el $20 \%$ del contenido.

Textos. En esta sección se publican reseñas, traducciones y memorias de eventos relacionados con las publicaciones en Arquitectura y Urbanismo.
A Frecuencia de publicación

Desde 1999 y hasta el 2015, la Revista de Arquitectura (Bogotá) publicó un volumen al año, a partir del 2016 se publicarán dos números por año en periodo anticipado, enero-junio y julio-diciembre, pero también maneja la publicación anticipada en línea de los artículos aceptados (versión Post-print del autor).

La Revista de Arquitectura (Bogotá) se divulga mediante versiones digitales (PDF, HTML, EPUB, XML) e impresascon un tiraje de 700 ejemplares, los tiempos de producción de estas versiones dependerán de los cronogramas establecidos por la editorial.

Los tiempos de recepción-revisión-aceptación pueden tardar entre seis y doce meses dependiendo del flujo editorial de cada sección y del proceso de revisión y edición adelantado.

Con el usuario y contraseña asignados, los autores pueden ingresar a la plataforma de gestión editorial y verificar el estado de revisión, edición o publicación del artículo.
(A) Canje

La Revista de Arquitectura (Bogotá) está interesada en establecer canje con publicaciones académicas, profesionales o científicas del área de Arquitectura y Urbanismo, como medio de reconocimiento y discusión de la producción científica en el campo de acción de la publicación.

Mecanismo

Para establecer canje por favor descargar, diligenciar y enviar el formato: RevArq FP20 Canjes

Universidad Católica de Colombia
(202I, enero-junio). Revista de
Arquitectura (Bogotá), 23(I),
I-I24. Doi: 10.14718

ISSN: I657-0308
E-ISSN: 2357-626X
Especificaciones:
Formato: $34 \times 24 \mathrm{~cm}$
Papel: Mate II5 g
Tintas: Negro y policromía

Contacto

Dirección postal:

Avenida Caracas No. 46-72

Universidad Católica de Colombia

Bogotá D. C., Colombia

Código postal: 111311

Facultad de Diseño

Centro de Investigaciones (CIFAR)

Sede El Claustro. Bloque "L", 4 piso

Diag. 46A No. 15b-10

Editor, Arq. César Eligio-Triana

Teléfonos:

+57 (1) $3277300-3277333$

Ext. 3109; 3112 o 5146

Fax: +57 (1) 2858895
Correo electrónico:

revistadearquitectura@ucatolica.edu.co cifar@ucatolica.edu.co

Página WEB:

www.ucatolica.edu.co

Vínculo Revistas científicas

http://publicaciones.ucatolica.edu.co revistas-cientificas

https://revistadearquitectura.ucatolica.edu.co/ 
Reflexiones en torno a la enseñanza de la arquitectura y el urbanismo en Colombia. Conversaciones con Juan Carlos

m. Pérgolis Valsecchi, René Carrasco Rey y Juan Carlos del

¿ Castillo

Reflections on the teaching of architecture and urban planning in Colombia. Conversations with Juan Carlos Pérgolis Valsecchi, René Carrasco Rey y Juan Carlos del Castillo

웅

La experiencia de caminar en ciudades latinoamericanas

The experience of walking in Latin American cities

Movilidad urbana de la población en la ciudad de Encarnación * Paraguay. Desarrollo urbano y gestión ambiental

$m$ Urban mobility of the population in the city of Encarnación, Paraguay.

这

ษั

La vivienda adecuada financiada según el ingreso.

El discurso de las Naciones Unidas

பं

La arquitectura frente a las innovaciones pedagógicas.

Pervivencia y resignificación de la Escuela Nueva en el Cono Sur Architecture facing the pedagogical innovations. Survival and resignification of the New School in the Southern Cone

Aporte de las competencias investigativas a la integración de saberes curriculares.

Una visión en el escenario de aprendizaje del diseño

arquitectónico

Contribution of research competencies to the integration of curricula knowledge. A vision in the architectural design learning scenario

Adobe como saber ancestral usado en construcciones

N autóctonas de Pore y Nunchía, Casanare (Colombia)

j. Adobe as an ancestral knowledge used in autochthonous constructions $\stackrel{4}{\longleftarrow}$

Paisaje construido y sustentabilidad urbana: huellas

$œ$

El Plan de Transformación de Osorno

Built landscape and urban sustainability: Identity prints of the modern landscape. The Osorno Transformation Plan

Indicadores de sostenibilidad social y su relación con el

$\hat{a}$

ن்

노 caso de estudio en Cuenca (Ecuador)

ن

Confort térmico en vivienda social multifamiliar de clima cálido en Colombia

느 Thermal comfort in multi-family social housing in a warm climate in ن் Colombia
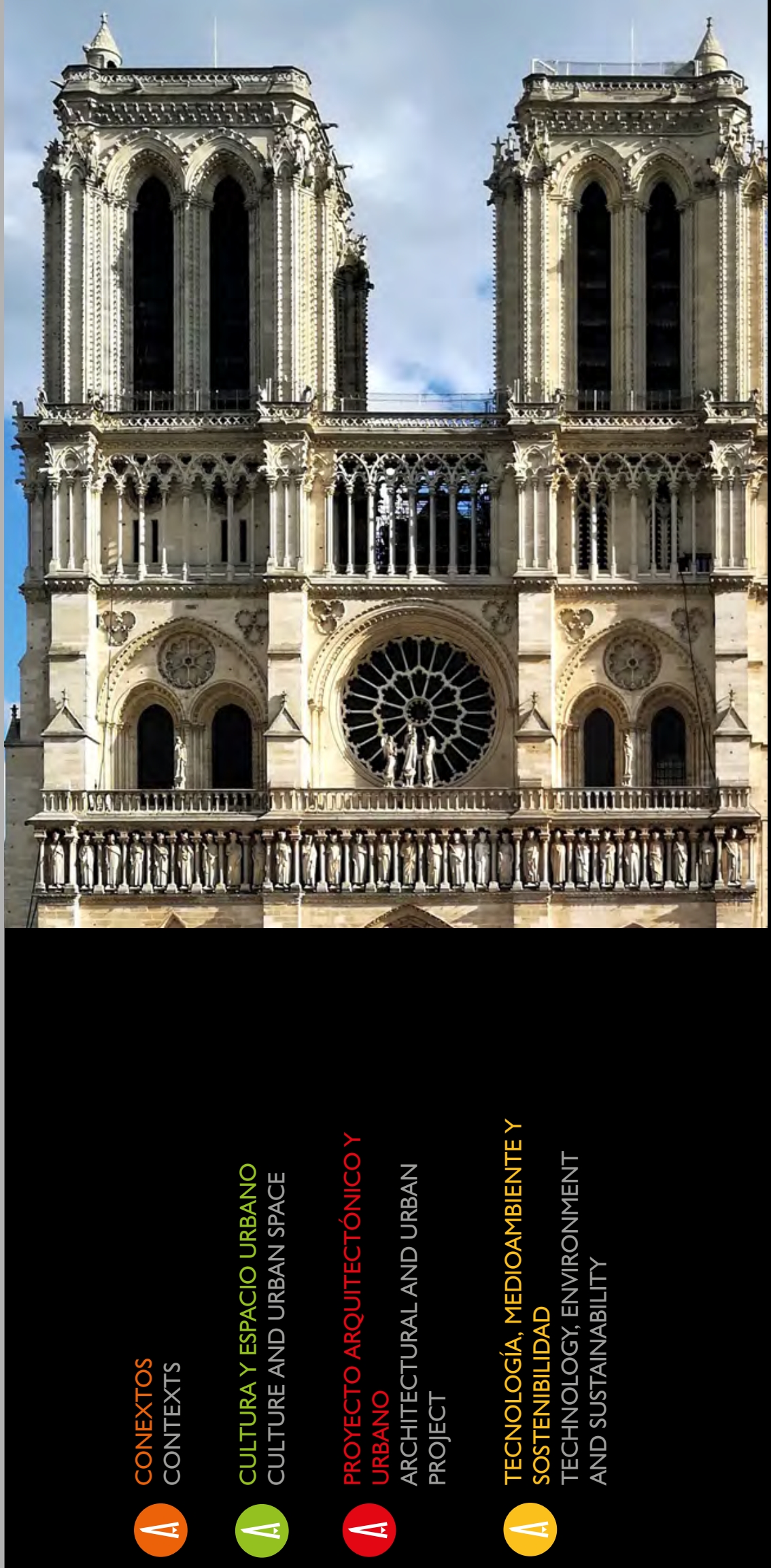

La Revista de Arquitectura es de acceso abierto, arbitrada e indexada y está presente en:
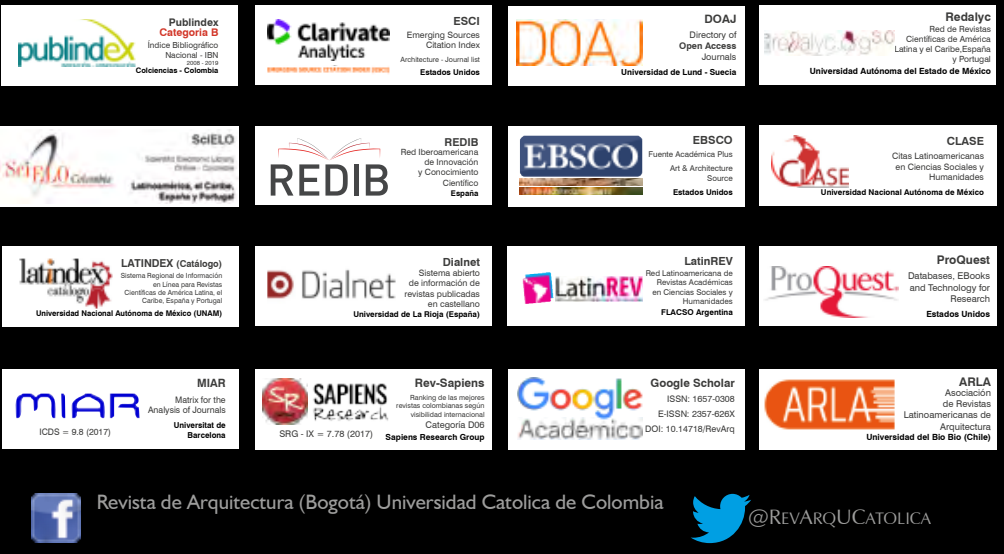

8
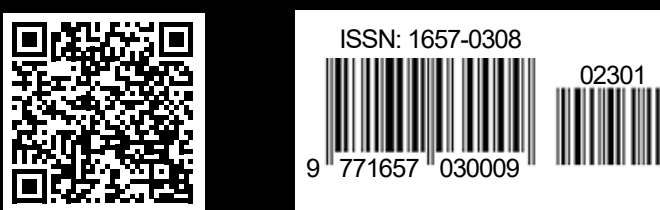